\title{
EFEITO DO USO DAS MÍDIAS SOCIAIS E DAS CAPACIDADES DINÂMICAS NO DESEMPENHO MERCADOLÓGICO DE MICRO, PEQUENAS E MÉDIAS EMPRESAS
}

\author{
${ }^{1}$ Silvia Spagnol Simi dos Santos \\ ${ }^{2}$ Sérgio Begnini \\ ${ }^{3}$ Carlos Eduardo Carvalho
}

Objetivo: Este artigo analisa o impacto da relação entre o uso das mídias sociais, das capacidades dinâmicas, e do desempenho mercadológico nas empresas, bem como o papel mediador das capacidades dinâmicas na relação entre o uso das mídias sociais e o desempenho mercadológico.

Método: A metodologia adotada foi quantitativa e descritiva, e a coleta de dados ocorreu por meio de um survey. Os resultados foram obtidos com base em uma amostra de 143 empresas. Realizou-se análise fatorial por meio do software SPSS ${ }^{\circledR}$, e a modelagem de equações estruturais por meio do software $\operatorname{AMOS}^{\circledR}$.

Originalidade/Relevância: Este estudo aprofunda o entendimento sobre o papel das mídias sociais em ambientes turbulentos, e sua relação com as capacidades dinâmicas e com o desempenho mercadológico, em micro, pequenas e médias empresas.

Resultados: As hipóteses foram testadas e apontaram para o efeito positivo do uso de mídias sociais nas capacidades dinâmicas, bem como do uso de mídias sociais no desempenho mercadológico. Porém, as capacidades dinâmicas não mediaram a relação entre o uso das mídias sociais e o desempenho mercadológico.

Contribuições teóricas/metodológicas: Os resultados contribuem para a ciência ao destacar o papel das mídias sociais em ambientes turbulentos, e sua relação com as capacidades dinâmicas e com o desempenho mercadológico, em micro, pequenas e médias empresas. Também contribuem teórica e empiricamente, na medida em que identificam fatores que permitem maximizar a produtividade das organizações.

Contribuições sociais/para a gestão: O maior desafio para os gestores de micro, pequenas e médias empresas é transformar os dados das mídias sociais em conhecimentos pessoais valiosos, sendo que explorar esses insights para criar valor agregado é a maior barreira de tais empresas.

Palavras-chave: Desempenho mercadológico. Capacidades dinâmicas. Mídias sociais. Micro, pequenas e médias empresas.

\section{Como citar:}

Santos, S. S. S. dos, Begnini, S., \& Carvalho, C. E. (2020). O efeito do uso das mídias sociais e das capacidades dinâmicas no desempenho mercadológico de micro, pequenas e médias empresas. Revista Brasileira de Marketing, 19(1), 174-196. https://doi.org/10.5585/remark.v19i1.17346. 


\section{Introdução}

O propósito deste estudo foi examinar a abordagem das Capacidades Dinâmicas (CD) na gestão estratégica, com foco em processos relacionados ao uso das Mídias Sociais (MS), no que tange ao Desempenho Mercadológico (DM) das firmas. As mídias sociais são sites criados na internet que permitem a elaboração colaborativa de conteúdo, o compartilhamento em vários formatos de informações e, ainda, a interação social (Telles, 2011), ou seja, uma empresa estar nas mídias sociais lhe permite criar relacionamentos e conversações.

Em ambientes dinâmicos (Eisenhardt \& Martin, 2000) como o das mídias sociais, nas quais é possível adquirir e gerar recursos em tempo real, as CD consistem em processos de tomada de decisão estratégica, que manipulam esses recursos para a obtenção de valor. Teece, Pisano e Shuen (1997; 2014) afirmam que as CD são essenciais, sobretudo em ambientes que mudam rapidamente, e Eisenhardt e Martin (2000) observam que isso varia de acordo com o dinamismo do mercado. Evidentemente, empresas que operam em ambientes dinâmicos de negócios são constantemente desafiadas a se adaptar a novas condições, existindo diversas formas de combinar capacidades dinâmicas com mudanças internas para levar as empresas a atingir alto desempenho no seu modelo de negócio (Jantunen, Tarkiainen, Chari, \& Oghazi, 2018)

As micro, pequenas e médias empresas (MPMEs) destacam-se no cenário brasileiro pela sua contribuição para a geração de empregos e aumento da riqueza do país, contribuindo para a economia nacional de forma surpreendente, tendo alcançado, no ano de 2015, mais de 6,8 milhões de estabelecimentos (Sebrae, 2017). A internet tem facilitado às empresas a obtenção de informações precisas sobre os clientes, os mercados, e os concorrentes (Wardaya, Idris, So \& Bandur, 2019), enfim, todos os seus stakeholders, sendo hoje imprescindível para o marketing.

O Brasil está entre os países com maior número de usuários de mídias sociais, com uma população ativa de 126,3 milhões de usuários; em 2017, a internet chegou a 74,9\% dos domicílios do Brasil (IBGE, 2019). As mídias sociais têm sido cada vez mais utilizadas pelas empresas como novas maneiras de conectar, comunicar e se envolver com uma ampla gama de partes interessadas (Mention, Barlatier, \& Josserand, 2019). Nesse conjunto de empresas, a imersão nas MS acaba sendo um diferencial, especialmente para os que desejam melhorar seu desempenho mercadológico, pois elas trazem dinamismo ao ambiente corporativo. Sendo inúmeras as estratégias e táticas que as mídias sociais oferecem, a questão central desta pesquisa pode ser resumida na seguinte pergunta: Qual é a relação entre mídias sociais, capacidades dinâmicas e desempenho mercadológico em micro, pequenas e médias empresas? Assim, o objetivo foi identificar o impacto da relação do uso das mídias sociais nas capacidades dinâmicas e no desempenho mercadológico, bem como o papel mediador das capacidades dinâmicas na relação entre o uso de MS e o DM. 
Foram identificados estudos de temática próxima a esta, como o de Wamba et al. (2017), que confirmou impactos diretos e indiretos do uso de ferramentas de big data sobre o desempenho das firmas, sendo que as capacidades dinâmicas tiveram um papel mediador no aumento do conhecimento e, com isso, melhoraram o desempenho. Wang \& Kim (2017) investigaram o uso das mídias sociais sob a perspectiva das capacidades organizacionais dinâmicas. Saavedra, Andreu e Criado (2016) afirmam que as empresas devem usar as redes sociais online de maneira intensiva, para potencializar a relação entre a orientação para o mercado e o desempenho. Wardaya et al. (2019) testaram a influência das capacidades dinâmicas no desempenho e na capacidade de marketing digital das empresas, bem como o papel do marketing digital.

Tais argumentos foram testados com dados primários coletados no ano de 2017, provenientes de uma amostra de 143 empresas. A coleta de dados (survey) foi realizada pela internet, mediante um formulário direcionado por e-mail aos gestores dessas firmas. Para testar os resultados utilizamos a modelagem de equações estruturais. Os resultados do estudo fornecem evidências de que existe um efeito positivo entre o uso de MS e o DM, e entre as CD e o DM. No entanto, as CD não mediaram o relacionamento entre MS e DM.

O artigo está dividido em cinco seções. A seção 2 apresenta uma revisão da literatura sobre MS, CD, e DM, além da construção das hipóteses. A seção 3 mostra os procedimentos metodológicos, e a seção 4 apresenta a análise e discussão dos resultados. A conclusão é apresentada na seção 5, seguida das limitações e sugestões de pesquisas futuras, na seção 6 .

\section{Revisão da literatura e hipóteses}

Capacidades Dinâmicas são entendidas como as estratégias desenvolvidas pela empresa, por meio das quais os gestores acessam os recursos, seja por aquisição ou criação própria, e os alocam, gerando novas estratégias e criando valor para a organização (Eisenhardt \& Martin, 2014), possibilitando desenvolver diferenciais de mercado frente à concorrência. Assim, os gestores têm maiores possibilidades para integrar, construir e reconfigurar, tanto as competências internas quanto as competências externas da organização. As capacidades dinâmicas constituem-se então, em fontes de vantagem competitiva sustentável para as organizações (Eisenhardt \& Martin, 2014).

As CD possibilitam que a empresa, atuando em ambientes complexos e de rápidas mudanças, possa detectar, analisar e compreender as fontes e os procedimentos que envolvem a criação e apropriação de riquezas (Teece et al., 2014). O estudo das CD auxilia a entender o como as empresas agem para reconfigurar seus recursos, em um ambiente de incertezas, ou mesmo em ambientes tranquilos, buscando elevado desempenho mercadológico. Assim, a relação das CD com a vantagem competitiva está fundamentada na possibilidade de a empresa criar, configurar, integrar 
e combinar seus recursos (Zollo \& Winter, 2002; Wang \& Ahmed, 2007; Eisenhardt \& Martin, 2014).

As CD mostram que a competência das empresas para mudar e reorganizar novos recursos, sob diferentes configurações, é mais relevante do que os próprios recursos, para a geração da vantagem competitiva sustentável (Teece et al., 2014); portanto, as CD alteram também a base de recursos das organizações por intermédio de estratégias organizacionais. Teece et al. (2014) destacam que a estratégia empresarial fornece uma vantagem competitiva, sustentada pelo desempenho da organização por meio das várias rotinas organizacionais desempenhadas pelas pessoas; conforme aumenta o número dessas rotinas, é possível desenvolver competências organizacionais. A competência organizacional existe quando as pessoas passam a ter consciência do resultado gerado por suas ações no desempenho geral da organização, por meio dos recursos que utilizam (Zollo \& Winter, 2002).

É com base no conjunto de recursos disponíveis e na sua reconfiguração que a empresa poderá alcançar resultados positivos. Seja com base no posicionamento estratégico (Porter, 1980), ou na visão baseada em recursos (VBR) (Barney, 1991; Peteraf, 1993), ou ainda com fulcro nas CD (Teece et al., 1997; Eisenhardt \& Martin, 2000), o desempenho mercadológico das empresas é assunto de pesquisas e debates. A busca por resultados positivos é constante em todas as empresas, pois é com base no desempenho alcançado que haverá, ou não, sustentabilidade no mercado.

No contexto do uso das mídias sociais por empresas, a fim de alcançar desempenho no seu mercado de atuação, vislumbram-se as capacidades dinâmicas como forças motrizes no processo, pois podem permitir que empresas, em um mercado em crescimento, identifiquem sinais importantes, avaliem novos processos, serviços ou produtos, e respondam às mudanças desse mercado (Wardaya et al., 2019). As mídias sociais modificaram a forma como uma organização se conecta, levando a diferentes possibilidades em várias funções organizacionais, do marketing ao gerenciamento de operações e ao desenvolvimento de novos produtos (Lam, Yeung, \& Cheng, 2016). Portanto, a imersão de empresas nas MS pode mudar a configuração de como elas se relacionam com seus stakeholders. Isso acontece porque as redes sociais fornecem uma plataforma para compartilhamento de experiências, acumulação de conhecimento e aprendizado organizacional (Nguyen, Yu, Melewar, \& Chen, 2015).

\subsection{Uso de ferramentas de mídias sociais, capacidades dinâmicas e desempenho mercadológico em micro, pequenas e médias empresas}

Pesquisas contemporâneas têm estudado o uso das mídias sociais, o desempenho mercadológico e as capacidades dinâmicas; no entanto, a literatura é escassa quanto à relação entre essas variáveis. Gupta e George (2016) identificaram vários recursos que, combinados, constroem 
capacidade de Big Data Analytics, e criaram um instrumento para medir tal capacidade e o desempenho da empresa. Lam et al. (2016) examinaram o impacto das iniciativas de mídia social sobre a eficiência operacional e a capacidade de inovação das empresas. Wang e Kim (2017) examinaram como o uso de mídias sociais pode ajudar as empresas a criar novas capacidades de gestão de relacionamento do consumidor, na busca por melhorar o desempenho de negócios. Wamba et al. (2017) examinaram os efeitos da capacidade analítica de big data sobre o desempenho da empresa, e também os efeitos mediadores das capacidades dinâmicas orientadas na relação entre capacidade analítica de big data e o desempenho da empresa. Ahani, Rahim e Nilashi (2017) buscaram os elementos preditores da adoção da estratégia de gestão de relacionamento com o cliente, em pequenas e médias empresas.

Outro estudo investigou como os empreendedores das MPMEs com capacidades inadequadas e recursos limitados transformaram o comércio eletrônico transfronteiriço na China (Li, Su, Zhang, \& Mao, 2017). O estudo revelou etapas-chave no processo, incluindo "renovação de cognição gerencial, desenvolvimento de redes sociais gerenciais, construção de equipe de negócios, e criação de capacidade organizacional” (Li et al., 2017, p. 21).

Braojos-Gomez, Benitez-Amado e Llorens-Montes (2015) pesquisaram os mecanismos fundamentais através dos quais as pequenas empresas aprendem a desenvolver competência em redes sociais, e concluíram que eles se valem da capacidade da infraestrutura de TI, da pressão do concorrente social, do gerenciamento de marketing, e da gestão da inovação. A capacidade tecnológica, a capacidade dos processos gerenciais e a capacidade organizacional estão diretamente ligadas à mudança tecnológica da empresa (Teece et al., 2014), e essa relação têm impacto sobre a criação de riqueza. Inseridas em ambientes de mudanças cada vez mais rápidas e frequentes, envolvendo principalmente as mídias sociais, as MPMEs precisam alcançar e manter o desempenho mercadológico. Uma possibilidade é desenvolver capacidades dinâmicas por meio das mídias sociais, a fim de elaborar e implementar a melhor estratégia ou prática empresarial. As CD exploram as competências internas e externas da empresa para enfrentar o ambiente turbulento e de mudanças (Teece et al., 2014). Com isso, elaboramos a primeira hipótese:

Hipótese 1: O uso de mídias sociais influencia positivamente as capacidades dinâmicas de MPMEs.

Estudos sobre mídias sociais e capacidades dinâmicas envolvem uma série de aspectos relevantes para as áreas de business e marketing. Zavattaro e Daspit (2016) abordaram questões relativas à inovação, enquanto Pogrebnyakov (2017) e Pinho e Prange (2016) focalizaram aspectos da internacionalização das empresas. Braganza, Brooks, Nepelski, Ali e Moro (2017) estudaram o gerenciamento; Braojos-Gomez et al. (2015) e Wieneke e Lehrer (2016) focaram no retorno de ativos; Liu, Deng, Hu e Chen (2015) e Erevelles, Fukawa e Swayne (2016) abordaram o marketing 
e a orientação para o mercado. O estudo de Wieneke e Lehrer (2016) relacionou as capacidades exigidas das empresas para transformar os dados das MS em conhecimentos valiosos, e explorar esses insights para criar valor agregado para os clientes. Wang e Kim (2017) exploraram o uso das MS no contexto do marketing $B 2 B$, a partir de uma perspectiva de capacidade organizacional dinâmica.

Lam et al. (2016) estudaram as iniciativas de mídias sociais enquanto impactos positivos sobre a eficiência operacional e a inovação. Assim, o uso de MS pode tornar-se uma fonte decisiva de desempenho mercadológico, pois já vivemos numa cultura de convergência (Jenkins, 2006). Essa cultura transforma a relação das empresas com fornecedores, funcionários e compradores, e ainda impacta os processos produtivos, a relação com outras empresas, e a avaliação da sociedade (Castells, 2003), e de modo mais forte o mercado consumidor. Por outro lado, o mau uso das MS também acarreta resultados negativos no desempenho das empresas. Assim, elaboramos a segunda hipótese de pesquisa:

Hipótese 2: O uso de mídias sociais influencia positivamente o desempenho mercadológico de MPMEs.

\subsection{Influência das capacidades dinâmicas no desempenho mercadológico}

Diante da concorrência, do ambiente complexo, e das incertezas que se estabelecem no mercado, as empresas necessitam de elementos que lhe possibilitem obter vantagem competitiva sustentada, e, desta forma, atingir elevado desempenho (Martynow \& Shafti, 2016). Um dos fatores que afetam diretamente o desempenho no mercado é a maturidade da organização, fruto da sua trajetória e de constantes revisões e melhorias dos processos internos (Barra \& Ladeira, 2017). Com isso, a implantação de estratégias diferenciadas, com base em capacidades e recursos raros, inimitáveis, de valor, e organizados, a empresa poderá gerar valor ao cliente e obter desempenho superior no mercado (Barney \& Hesterly, 2007).

Utilizando alguns dados e informações, é possível identificar e mensurar o DM das empresas. Tentando realizar tal trabalho, alguns autores vêm, ao longo do tempo, identificando e utilizando diferentes variáveis: satisfação dos clientes, satisfação dos funcionários, lucratividade, crescimento das vendas, e reputação da empresa (Venkatraman \& Ramanujam, 1986); capacidade de produção, evolução no número de clientes, fidelização dos clientes, investimento em treinamentos, propaganda e modernização, qualidade do produto/serviço, valor da marca, volume de vendas (Callado, Callado, \& Andrade, 2008); volume de vendas e tomada de decisão (Lombardi, Bertoni, Nozawa, Bellato, \& Grandi, 2010); fidelização dos clientes, atração de novos clientes, volume de vendas, market share, e satisfação dos clientes (Santos, Bronzo, Oliveira, \& Resende, 2014). 
Em estudo anterior, Wardaya et al. (2019) testaram a influência da capacidade dinâmica no desempenho de firmas da Indonésia, com resultados positivos. Para os autores, a capacidade dinâmica aumenta a velocidade e eficiência das respostas das pequenas e médias empresas à turbulência ambiental, o que, por sua vez, melhora o desempenho. Percebe-se que para atingir um DM favorável, as empresas necessitam desenvolver $\mathrm{CD}$, isto é, aprender a se reinventar constantemente. Precisam ser capazes de atrair novos clientes, reter os atuais, aumentar a lucratividade, bem como a participação no mercado, além de oferecer maior qualidade, preços atraentes e atendimento personalizado (Christensen, 1997; OECD, 2005; Tidd, Bessant, \& Pavitt, 2005; Tigre, 2006; Chesbrough, 2012). Ao desenvolver CD, as empresas estarão mais preparadas para competir e atuar no mercado, o que lhes possibilita alcançar maior desempenho mercadológico. Assim, elaboramos a terceira hipótese da pesquisa:

Hipótese 3: As capacidades dinâmicas influenciam positivamente o desempenho mercadológico de MPMEs.

\subsection{O efeito das Capacidades Dinâmicas}

Poucos estudos foram encontrados a respeito do efeito de mediação das capacidades dinâmicas na relação entre o uso de mídias sociais e desempenho mercadológico. Saavedra et al. (2016) concluíram que as empresas devem usar as redes sociais online de maneira intensiva para potencializar a relação entre a orientação para o mercado e o desempenho. Se as empresas participam ativamente das redes sociais online e possuem, realmente, uma estratégia para o marketing de mídia social, investindo intensamente em seu uso, além de um gerente ou uma agência de marketing digital, elas podem aproveitar o potencial dessas redes como uma ferramenta de marketing. Os resultados mostram que não é suficiente ter alguns perfis e executar algumas ações básicas do marketing de mídia social, sendo necessário um esforço forte e dedicado nesta forma de marketing para observar um impacto real no desempenho.

O estudo de Wamba et al. (2017) é semelhante ao nosso, e demonstrou haver uma relação entre o uso de ferramentas relacionadas às capacidades de big data e o desempenho, e também entre aquelas e as capacidades dinâmicas das firmas; além disso, mostrou que as CD medeiam a relação entre capacidade de big data e desempenho. Apesar de tratar do uso das informações geradas pelas mídias sociais por meio de big data, e nosso estudo ter como foco o uso de mídias sociais, especificamente, acredita-se que os achados de Wamba et al. (2017) podem contribuir para esta investigação, em especial porque o mesmo confirma impactos diretos e indiretos de big data sobre o desempenho das firmas, sendo que as capacidades dinâmicas têm um papel mediador no aumento de conhecimentos e, assim, melhoram o desempenho. 
Wardaya et al. (2019) estudaram gerentes de pequenas e médias empresas da Indonésia, utilizando um modelo próximo ao desta pesquisa, e testaram a influência das capacidades dinâmicas no desempenho e na capacidade de marketing digital das empresas, bem como o inverso, ou seja, a influência da capacidade de marketing digital sobre o desempenho das firmas. Testaram, ainda, o efeito mediador do marketing digital nesta relação, com resultados positivos.

A literatura é escassa quanto ao efeito mediador das Capacidades Dinâmicas na relação entre o uso de mídias sociais por empresas e seu desempenho mercadológico, portanto cabe pesquisar este aspecto. Com isso, surge a quarta hipótese:

H4: As capacidades dinâmicas medeiam a relação entre o uso de ferramentas das mídias sociais e o desempenho mercadológico de MPMEs.

A Figura 1 apresenta o modelo conceitual da pesquisa, mostrando a influência mútua dos constructos.

Figura 1 - Modelo conceitual

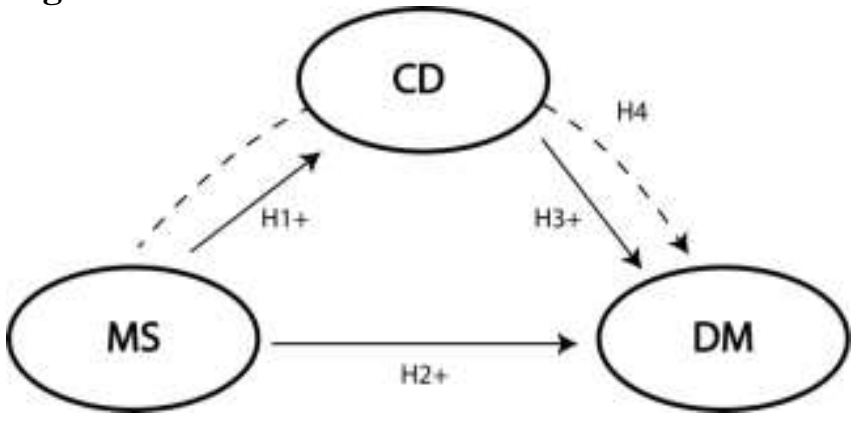

Fonte: Os autores, 2018.

\section{Método}

O objetivo principal desta pesquisa foi entender a relação entre mídias sociais, capacidades dinâmicas, e o desempenho mercadológico. $\mathrm{O}$ foco foram as micro, pequenas e médias empresas da indústria, do comércio, e de serviços que atuam no estado de Santa Catarina. Os dados primários foram obtidos por meio de um survey, pela aplicação de um questionário direcionado aos gestores das empresas. Entendeu-se que, ao estudar o maior número possível de empresas, haveria maior possibilidade de contribuir para a literatura. 


\subsection{Dados e amostra}

Este estudo foi realizado com micro, pequenas e médias empresas que integram a Federação das Associações Empresariais de Santa Catarina, Brasil, que reúne mais de 35 mil empresas filiadas a 148 Associações Empresariais do Estado de Santa Catarina. Fizemos a caracterização da amostra segundo o Serviço Brasileiro de Apoio às Micro e Pequenas Empresas (Sebrae). Em algumas publicações do Sebrae sobre MPEs, o critério para definição deste segmento empresarial é o porte das empresas em termos de pessoal ocupado, portanto, assim foi feito. Foram obtidas 172 respostas, das quais 143 passaram a integrar a amostra do estudo, pois estavam completas e atendiam aos critérios da pesquisa (micro, pequenas e médias empresas). A pesquisa é descritiva e quantitativa, uma vez que buscou medir o efeito do uso das mídias sociais nas capacidades dinâmicas, e sua relação com o desempenho mercadológico.

\subsection{Elaboração e validação do instrumento}

Para construir e validar o instrumento de pesquisa, primeiramente elaboramos as assertivas, com base na literatura. Trata-se de dados primários, coletados por um questionário com 26 questões, aplicado por meio do Survey Monkey. Quatro questões foram de identificação das empresas e, uma referia-se a compreender o uso das mídias sociais, estruturada em escala Likert de 5 pontos, onde 1 significa "nenhum uso" e 5 "máximo uso". Outras 21 assertivas, mostradas na Tabela 1, foram elaboradas para que os respondentes escolhessem uma posição (pontuação) de 0 a 100, em uma barra contínua, onde 0 significava "reduziu $10 \%$ ou mais", 50 indicava "não reduziu nem aumentou" e 100 apontava que "aumentou 10\% ou mais". A Figura 2 refere-se a uma das assertivas do questionário, e mostra como elas foram organizadas. O questionário na íntegra consta do Apêndice 1.

Figura 2 - Esboço da formulação das assertivas

monitoramento das midias sociais da nossa empresa:

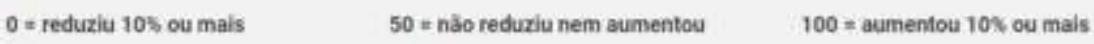

Fonte: Os autores, 2018.

A Tabela 1 mostra as variáveis latentes, as assertivas de cada variável latente, e os autores que as formularam. $\mathrm{O}$ instrumento resultante foi então submetido a um pré-teste com três profissionais e, posteriormente, validado por um especialista da área. Após a validação, o questionário foi enviado por e-mail para a população. Essas variáveis estabelecem indicadores importantes, pois criam a possibilidade de comparar e medir o desempenho das empresas. $\mathrm{O}$ 
questionário foi organizado em quatro partes: a primeira serviu para caracterizar o respondente; a segunda buscou identificar a utilização das MS pelas empresas; a terceira procurou identificar as CD presentes nas empresas; e a quarta buscou medir o DM de cada empresa.

Tabela 1 - Autores que formularam as assertivas de cada constructo

\begin{tabular}{|c|c|c|}
\hline Autores & Assertivas & $\begin{array}{c}\text { Constru } \\
\text { cto }\end{array}$ \\
\hline $\begin{array}{l}\text { Nguyen et al. (2015) } \\
\text { Braojos-Gomez et al. } \\
(2015) \\
\text { Wieneke \& Lehrer } \\
\text { (2016) } \\
\text { Lam et al. (2016) }\end{array}$ & $\begin{array}{l}\text { 1. Atuação da empresa nas MS } \\
\text { 2. Utilização de informações das MS para conhecer o cliente } \\
\text { 3. Monitoramento das MS } \\
\text { 4. Planejamento das estratégias da empresa para MS } \\
\text { 5. Interação com o consumidor por meio das MS } \\
\text { 6. Ações da empresa a partir dos resultados das MS } \\
\text { 7. Investimento da empresa em MS } \\
\text { 8. Divulgação dos produtos/serviços nas MS }\end{array}$ & MS \\
\hline $\begin{array}{l}\text { Teece et al. (1997) } \\
\text { Eisenhardt \& Martin } \\
\text { (2000) } \\
\text { Zollo \& Winter (2002) } \\
\text { Wang \& Ahmed (2007) } \\
\text { Lin \& Wu (2014) } \\
\text { Teece et al. (2014) } \\
\text { Eisenhardt (2014) } \\
\text { Braganza } \text { et al. (2017) }\end{array}$ & $\begin{array}{l}\text { 1. Trabalho para que os recursos de MS não fossem } \\
\text { identificados/imitados } \\
\text { 2. Compartilhamento das informações das MS entre setores da } \\
\text { empresa, para visão integrada } \\
\text { 3. Com o uso de MS, reorganização da equipe de negócios ou } \\
\text { competências internas } \\
\text { 4. Com o uso de MS, a capacitação da infraestrutura de TI } \\
\text { 5. Com o uso de MS, pressão do concorrente } \\
\text { 6. Com o uso de MS, esforço para o gerenciamento de MKT } \\
\text { 7. Com o uso de MS, esforço para a gestão da inovação } \\
\text { 8. Com o uso de MS, agilidade de resposta às mudanças do ambiente } \\
\text { competitivo }\end{array}$ & CD \\
\hline $\begin{array}{l}\text { Venkatraman \& } \\
\text { Ramanujam (1986) } \\
\text { Tidd } \text { et al. }(2005) \\
\text { Barney \& Hesterly } \\
\text { (2007) } \\
\text { Callado et al. }(2008) \\
\text { Lombardi } \text { et al. }(2010) \\
\text { Santos } \text { et al. }(2014) \\
\text { Liu } \text { et al. } \text { (2015) }\end{array}$ & $\begin{array}{l}\text { 1. Nível de satisfação dos clientes } \\
\text { 2. Nível de retenção dos clientes } \\
\text { 3. Número de novos clientes } \\
\text { 4. Faturamento da empresa } \\
\text { 5. Volume de vendas da empresa }\end{array}$ & $\mathrm{DM}$ \\
\hline
\end{tabular}

Fonte: Os autores, 2018.

Após a coleta de dados, procedeu-se ao tratamento por meio dos testes de KolmogorovSmirnov e Shapiro-Wilk para verificar a normalidade. Posteriormente, usamos a análise fatorial exploratória (AFE) para avaliar a dimensionalidade das escalas, usando o software SPSS®. Em seguida, realizamos a modelagem de equações estruturais (SEM), utilizando o software AMOS®. Também foi utilizada a macro PROCESS V3.4 Andrew F. Hayes, para conferir a existência de mediação entre as variáveis. Como variáveis de controle, foram testadas o tamanho das empresas e o setor. Para isto foram efetuadas análises de regressão e análise de variância - ANOVA, considerando como independentes as variáveis de controle; não foi localizada nenhuma influência destas sobre os construtos estudados. 


\section{Resultados}

Micro, pequenos e médios negócios empresariais no Brasil constituíram, no ano de 2015, uma população com mais de 6,8 milhões de estabelecimentos (Sebrae, 2017). Nesse conjunto empresarial, a imersão nas MS acaba sendo um diferencial, especialmente para os que desejam melhorar seu desempenho mercadológico. Para facilitar a análise dos dados referentes às MPMEs participantes desta pesquisa, realizamos, primeiramente, uma análise descritiva dos dados coletados, e em seguida, por meio da modelagem de equações estruturais, testamos as hipóteses formuladas.

\subsection{Análise descritiva dos dados}

$\mathrm{Na}$ amostra pesquisada (serviços, comércio e indústria), o setor de serviços possui o maior número de empresas, seguido pelo comércio e pela indústria. Com relação à atuação, destacam-se as microempresas que, nos três setores, somam $65,03 \%$, enquanto as pequenas são $29,37 \%$ e as médias $5,60 \%$. Por setor, a indústria, com 52,67\%, apresentou o maior número de funcionários, seguida pelos serviços, com $31,62 \%$, e pelo comércio, com $15,71 \%$. Ficou evidente que as empresas utilizam, de alguma forma, mídias sociais. Com base nas respostas, a Tabela 2 mostra as médias de utilização de cada mídia, por setor, e o geral.

Tabela 2 - Média da utilização das Mídias Sociais, por setor de atuação, e a média geral

\begin{tabular}{|c|c|c|c|c|c|c|c|c|c|c|c|c|c|}
\hline 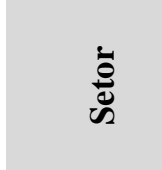 & 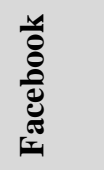 & 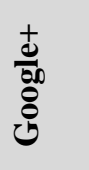 & 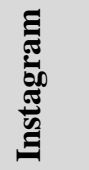 & 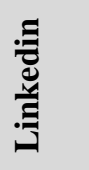 & 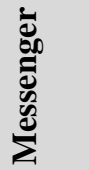 & 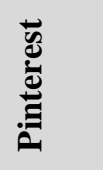 & 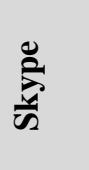 & 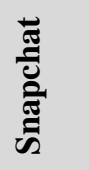 & 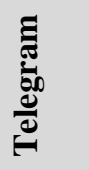 & $\begin{array}{l}\stackrel{\Xi}{\text { ह }} \\
\text { हE }\end{array}$ & 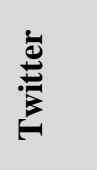 & 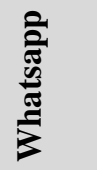 & $\begin{array}{l}\stackrel{\Xi}{\Xi} \\
\stackrel{\Xi}{\Xi}\end{array}$ \\
\hline Comércio & 4,03 & 2,12 & 2,88 & 1,50 & 2,41 & 1,12 & 2,15 & 1,24 & 1,12 & 1,09 & 1,50 & 4,38 & $\overline{1,79}$ \\
\hline Indústria & 3,16 & 1,89 & 1,95 & 2,42 & 1,89 & 1,00 & 3,00 & 1,00 & 1,00 & 1,00 & 1,21 & 3,95 & 1,79 \\
\hline Serviços & 3,77 & 2,21 & 2,62 & 1,97 & 2,28 & 1,31 & 2,57 & 1,20 & 1,12 & 1,01 & 1,47 & 4,38 & 2,00 \\
\hline Geral & 3,65 & 2,07 & 2,48 & 1,96 & 2,19 & 1,14 & 2,57 & 1,15 & 1,08 & 1,03 & 1,39 & 4,24 & 1,86 \\
\hline
\end{tabular}

Fonte: Dados da pesquisa, 2018.

Os respondentes selecionaram, entre 1 e 5, a resposta que melhor representava sua realidade, onde 1 significou "nenhum uso daquela mídia social" e 5 "máximo uso daquela mídia social”. A média geral para a utilização de cada mídia demonstra que a maior utilização é do Whatsapp, seguido pelo Facebook. E apresentam menor utilização o Tumblr, Telegram, Pinterest e Snapchat. Além de analisar a utilização das MS, também foi possível identificar alguns aspectos referentes a cada um dos três constructos da pesquisa.

O constructo "Mídias Sociais" [MS] foi identificado com oito assertivas, cujos resultados indicaram as médias das respostas acima de 50 pontos, mostrando que as MPMEs de Santa Catarina 
aumentaram o uso das mídias sociais em 2017, em relação ao ano anterior. O maior crescimento refere-se à divulgação dos produtos ou serviços nas MS, que obteve a maior média $(74,68)$. O menor refere-se ao trabalho para garantir que os recursos das MS não fossem facilmente identificados ou imitados pelos concorrentes, que teve média de 53,14.

O constructo "Capacidades Dinâmicas" [CD] abrangeu oito assertivas, e também atingiu média acima de 50 pontos. Isso indica que as empresas, de alguma forma, buscaram desenvolver ou reconfigurar competências internas e externas para adaptar-se às mudanças (Teece et al., 1997) e alcançar vantagem competitiva (Lin \& Wu, 2014). A agilidade de resposta às mudanças do ambiente competitivo, usando as mídias sociais, foi a variável com maior média $(70,66)$. A menor média $(51,13)$ refere-se ao uso das mídias sociais na capacidade de infraestrutura de TI.

O terceiro constructo, "Desempenho Mercadológico" [DM], envolveu cinco assertivas e, na média, indica que as MPMEs conseguiram alcançar resultados positivos. Entender o DM é importante, pois auxilia as MPMEs a identificar ameaças e oportunidades, a manter atenção às mudanças no mercado (Chenhall \& Moers, 2015), e a visualizar as oportunidades de inovação do negócio. Em relação ao ano de 2016, o maior crescimento foi em relação ao nível de satisfação dos clientes, com média de 70,73, e o menor crescimento foi do nível de retenção dos clientes, com média de 63,10. A Tabela 3 apresenta os valores descritivos de cada indicador.

Tabela 3 - Variáveis e descrição dos dados da pesquisa

\begin{tabular}{|c|c|c|c|c|c|c|}
\hline Constructo & Variáveis & $\mathbf{M}$ & DP & Variação & Assimetria & Curtose \\
\hline \multirow{8}{*}{$\begin{array}{l}\text { Mídias } \\
\text { Sociais (MS) }\end{array}$} & Atuação da empresa nas MS & 72,31 & 26,31 & 692,39 &,- 871 & ,268 \\
\hline & $\begin{array}{l}\text { Utilização de informações das MS para } \\
\text { conhecer o cliente }\end{array}$ & 66,65 & 24,04 & 577,93 &,- 188 &,- 515 \\
\hline & Monitoramento das MS & 69,83 & 26,08 & 679,92 &,- 709 & , 195 \\
\hline & $\begin{array}{l}\text { Planejamento das estratégias da empresa para } \\
\text { MS }\end{array}$ & 69,06 & 25,54 & 652,23 &,- 605 & ,103 \\
\hline & Interação com o consumidor por meio das MS & 74,07 & 25,18 & 634,02 &,- 838 & ,369 \\
\hline & $\begin{array}{l}\text { Ações da empresa a partir dos resultados das } \\
\text { MS }\end{array}$ & 64,91 & 24,91 & 620,51 &,- 358 &,- 138 \\
\hline & Investimento da empresa em MS & 62,49 & 27,17 & 738,24 &,- 352 &,- 184 \\
\hline & Divulgação dos prod./serv. nas MS & 74,68 & 25,66 & 658,60 &,- 930 & ,407 \\
\hline \multirow{7}{*}{$\begin{array}{l}\text { Capacidades } \\
\text { Dinâmicas } \\
\text { (CD) }\end{array}$} & $\begin{array}{l}\text { Trabalho para que os recursos de MS não } \\
\text { fossem identificados/imitados }\end{array}$ & 53,14 & 25,26 & 638,16 &,- 012 & ,242 \\
\hline & $\begin{array}{l}\text { Compartilhamento das informações das MS } \\
\text { entre setores da empresa, para visão integrada }\end{array}$ & 59,19 & 28,49 & 811,59 &,- 281 &,- 341 \\
\hline & $\begin{array}{l}\text { Com o uso de MS, reorganização da equipe de } \\
\text { negócios ou competências internas }\end{array}$ & 57,52 & 24,61 & 605,50 &,- 177 & ,423 \\
\hline & $\begin{array}{l}\text { Com o uso de MS, a capacitação da } \\
\text { infraestrutura de TI }\end{array}$ & 51,13 & 23,25 & 540,34 &,- 217 & ,966 \\
\hline & Com o uso de MS, pressão do concorrente & 58,57 & 22,71 & 515,87 &,- 120 & ,731 \\
\hline & $\begin{array}{l}\text { Com o uso de MS, esforço para o } \\
\text { gerenciamento de MKT }\end{array}$ & 65,89 & 27,10 & 734,58 &,- 657 & ,204 \\
\hline & $\begin{array}{l}\text { Com o uso de MS, esforço para a gestão da } \\
\text { inovação }\end{array}$ & 67,10 & 24,99 & 624,70 &,- 472 & , 152 \\
\hline
\end{tabular}


Com o uso de MS, agilidade de resposta às mudanças do ambiente competitivo

Nível de satisfação dos clientes

Desempenho Nível de retenção dos clientes

Número de novos clientes

\begin{tabular}{rrrrr}
70,66 & 24,66 & 608,25 &,- 646 &, 201 \\
\hline 70,73 & 21,45 & 460,07 &,- 612 &, 679 \\
63,10 & 24,09 & 580,54 &,- 366 &, 272 \\
67,87 & 25,41 & 645,46 &,- 695 &, 466 \\
63,64 & 24,33 & 592,02 &,- 281 &, 334 \\
65,12 & 24,32 & 591,53 &,- 442 &, 415
\end{tabular}

co (DM) Faturamento da emp
Volume de vendas
Fonte: Dados da pesquisa, 2018.

\subsection{Análise exploratória dos dados}

Como os dados se aproximam da normalidade, com valores de curtose e assimetria relativamente baixos, antes de proceder ao teste de hipóteses, por meio da Modelagem de Equações Estruturais (SEM), optamos por realizar a análise fatorial exploratória (AFE), para avaliar a dimensionalidade das escalas. A AFE é utilizada em estudos com constructos reflexivos, reunindo as variáveis em um número de fatores ideal para melhor representar os dados (Hair, William, Babin, \& Anderson, 2009). Utilizamos o método de componentes principais e o critério eigenvalue igual a 1 (um), que permitiram extrair os fatores.

Para a correta adequação do método, as variáveis "Planejamento das estratégias da empresa para MS" e "Com o uso de MS, agilidade de resposta às mudanças do ambiente competitivo", precisaram ser excluídas. O grau de explicação do teste Kaiser-Meyer-Olkin (KMO) ficou em 0,894, e o valor do Qui-quadrado do teste de Bartlett foi de 1813,08, significante a 0,000, o que indica a adequação dos dados (Hair et al., 2009). A variância explicada foi de 67,05\%, atendendo aos valores apontados na literatura (Hair et al., 2009).

Em seguida, realizamos o teste de confiabilidade por meio do Alpha de Cronbach para os três constructos. Os valores do Alfa de Cronbach para os constructos MS, CD e DM foram, respectivamente, 0,912, 0,890, e 0,893. Assim, como recomendado por Hair et al. (2009), as medidas de consistência interna e de confiabilidade do modelo foram adequadas para prosseguir com a realização da Modelagem de Equações Estruturais.

\subsection{Modelagem de equações estruturais}

Com a realização da análise exploratória foi possível chegar ao modelo mais ajustado, composto por sete indicadores para o constructo $\mathrm{CD}$, sete para MS e cinco para DM, como mostra a Figura 3. 
Figura 3 - Variáveis e descrição dos dados da pesquisa

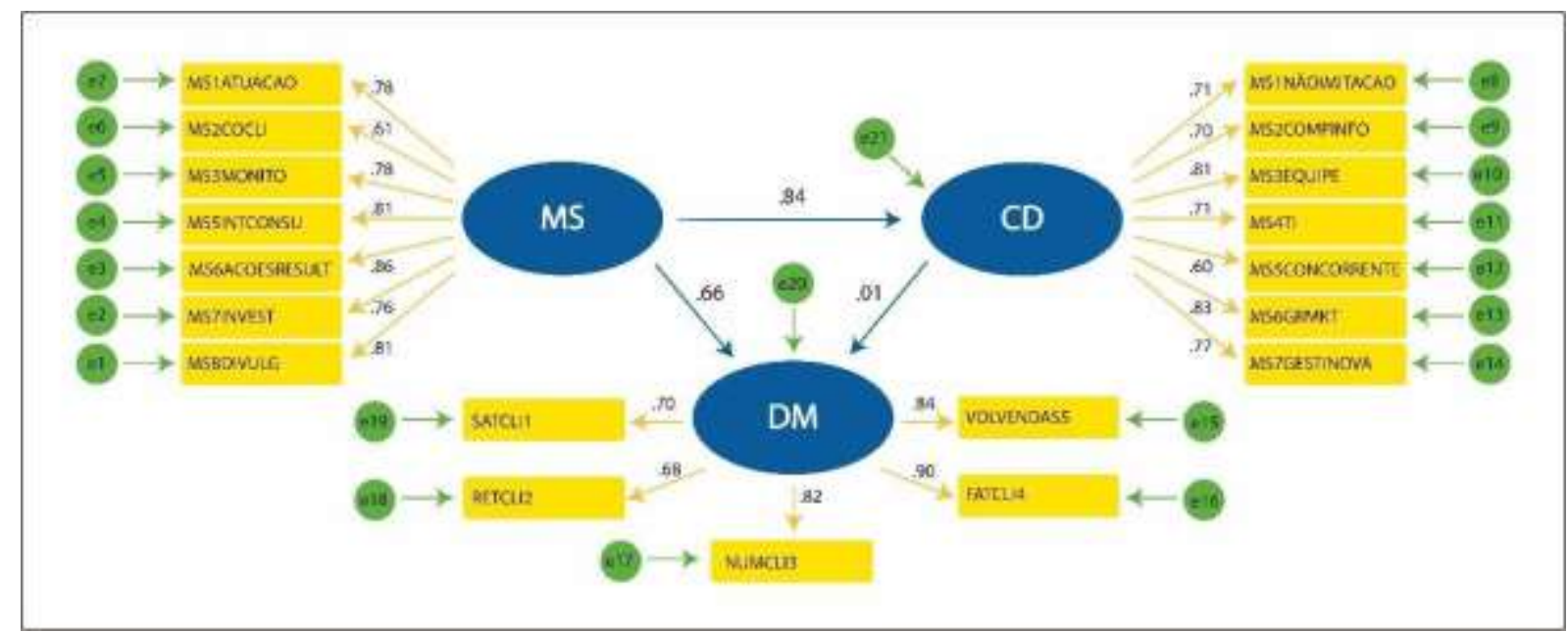

Fonte: Dados da pesquisa, 2018.

Os indicadores de ajuste geral do modelo foram 2,537 para o valor de CMIN/DF, 0,877 para o CFI, e 0,104 para o RMSEA, e mostram que o ajuste do modelo não é o ideal, mas é razoável, conforme o preconizado por Hair et al. (2009).

A última etapa consistiu em analisar as hipóteses de pesquisa e os testes de significância. Os coeficientes calculados para as relações entre os constructos previstos nas hipóteses H1 e H2 foram aceitos ( $p$-value=0,000). Assim, foi confirmado que MS influenciam CD e DM. Já a hipótese H3 foi rejeitada, mostrando que DM não sofre influência de $C D$, conforme indicado na Tabela 4 .

Tabela 4 - Resultados dos testes de significância dos modelos de pesquisa

\begin{tabular}{cccccccc}
\hline Hipótese & $\begin{array}{c}\text { Variável } \\
\text { Dependente }\end{array}$ & & $\begin{array}{c}\text { Variável } \\
\text { Independente }\end{array}$ & Estimativa & $\boldsymbol{p}$-value & $\boldsymbol{C . R}$. & Status \\
\hline H1 & MS & $--->$ & CD & .842 & 0,000 & 6.409 & Aceita \\
H2 & MS & $--->$ & DM & .657 & 0,000 & 3.646 & Aceita \\
H3 & CD & $--->$ & DM & .007 & 0.691 & 0.420 & Rejeitada \\
\hline
\end{tabular}

Fonte: Dados da pesquisa, 2018.

Para calcular a mediação foram utilizados os critérios indicados por Hair et al. (2009). Assim: (i) a variável independente e a variável dependente devem apresentar correlação significante. Neste caso, a correlação entre MS e DM foi de 0,624, com p-value 0,000, atendendo ao primeiro item; (ii) a variável independente deve apresentar correlação significante com a variável que está sendo testada como mediadora. Neste caso, a correlação entre MS e CD foi de 0,743, com p-value de 0,000, atendendo também ao segundo item; (iii) a variável mediadora deve apresentar correlação significante com a variável dependente. Neste teste, CD e DM apresentaram correlação de 0,528 com p-value de 0,000 atendendo ao terceiro item.

O próximo passo foi calcular a relação de efeito entre a variável independente e a dependente e, na sequência, calcular novamente com a inclusão da variável mediadora. $\mathrm{O}$ primeiro 
cálculo foi realizado por meio do software AMOS®, com a participação dos constructos MS e DM. $\mathrm{O}$ coeficiente de caminho foi 0,66 , significante ao nível 0,001 . Na sequência foi incluído o constructo $\mathrm{CD}$, e os cálculos foram novamente refeitos. O coeficiente de caminho entre MS e DM não se alterou, mantendo-se em 0,66, significante a nível 0,001, conforme apresentado na Figura 3. Hair et al. (2009) afirmam que se a relação entre a variável independente e a dependente continua significante e inalterada, com a inclusão da variável mediadora, então não há mediação. Assim, percebe-se que a mediação de CD entre MS e DM não foi sustentada.

Para confirmar a não existência da mediação, foi realizado um segundo cálculo com o auxílio da Macro Process para SPPS, de Andrew F. Hayes. Incluiu-se a variável independente MS, a variável dependente DM, e a variável mediadora CD. A Tabela 5 mostra o resultado.

Tabela 5 - Resultados dos testes de significância dos modelos de mediação

\begin{tabular}{|c|c|c|c|c|c|c|}
\hline \multicolumn{7}{|c|}{ Resumo do modelo } \\
\hline $\mathrm{R}$ & R-sq & MSE & $\mathrm{F}$ & df1 & df2 & $\mathrm{p}$ \\
\hline 0,631 & 0,398 & 245,253 & 46,310 & 2,000 & 140,000 & 0,000 \\
\hline \multicolumn{7}{|c|}{ Modelo } \\
\hline & coef & se & $\mathrm{t}$ & $\mathrm{p}$ & LLCI & ULCI \\
\hline Constante & 22,878 & 4,691 & 4,878 & 0,000 & 13,605 & 32,152 \\
\hline MS & 0,499 & 0,095 & 5,272 & 0,000 & 0,312 & 0,686 \\
\hline $\mathrm{CD}$ & 0,147 & 0,100 & 1,469 & 0,144 & $-0,051$ & 0,345 \\
\hline
\end{tabular}

Nota: Variável dependente: DM

Fonte: Dados da pesquisa, 2018.

Percebe-se que a explicação do modelo é de 39,8\%. Também mostra que MS explica 49,9\% de DM, significante a 0,000. Quando analisada a variável latente $\mathrm{CD}$, percebe-se que ela explica 14,7\% de DM. Contudo, tal explicação não é significante, é possível concluir que CD não contribui para a explicação do modelo. Desta forma, como o resultado anterior já apontava, H4 foi rejeitada, uma vez que CD não medeia a relação entre MS e DM.

\subsection{Discussão}

Os resultados deste estudo assinalam que as MS geram CD e também DM, nas MPMEs investigadas; contudo, quando as MS são mediadas pelas CD, não há influência no DM, ou seja, o uso das MS não gera DM de maneira indireta, por meio das CD. As empresas participantes deste estudo confirmam as considerações de Teece et al. (1997), de que as empresas exploram suas competências internas e externas para enfrentar o ambiente de mudança. Assim, as MS geram CD, o que ratifica a $\mathrm{H} 1$ proposta “ $M S$ gera $C D$ ” estatisticamente. Os resultados aproximam-se daqueles 
indicados por Lam et al. (2016), que consideraram que as mídias sociais possibilitam a melhoria das rotinas dinâmicas, conquistando melhor desempenho.

Existem indicações de que as empresas analisadas precisam internalizar o uso das mídias sociais para conhecer e se relacionar melhor com seus stakeholders. A imersão de empresas nas MS pode mudar sua configuração de relacionamento com os stakeholders, pois fornecem uma plataforma para compartilhamento de experiências, acumulação de conhecimento e aprendizado organizacional (Nguyen et al., 2015; Lam et al., 2016). As MPMEs participantes do estudo, no ano de 2017, aumentaram timidamente o compartilhamento de experiências, a utilização das informações para conhecer os clientes, e também as ações, a partir das informações das MS.

No constructo MS, as pequenas empresas possuem médias de atuação superior nas mídias sociais, seguidas pelas microempresas. Tal resultado aproxima-se daquele encontrado por BraojosGomez et al. (2015), que mostraram, em sua análise empírica, que a competência das redes sociais é mais importante para as empresas de menor tamanho. Uma explicação pode ser o fato de que, por possuírem menor portfólio de recursos financeiros, as capacidades de mídias tornam-se mais importantes para elas do que para empresas maiores.

Os resultados dos cálculos estatísticos sustentaram a hipótese H2 de que "a utilização de MS gera desempenho". Tal resultado reflete e confirma os resultados de Venkatraman e Ramanujam (1986) e de Callado et al. (2008), quanto à identificação e mensuração do uso das MS nas empresas para aumentar o desempenho. Os resultados desta pesquisa aproximam-se dos de Lombardi et al. (2010) e de Santos et al. (2014), quando estes indicam que as empresas atuam em ambientes competitivos e de incertezas, e que as decisões tomadas pelos gestores possibilitam atingir maior lucratividade, crescimento das vendas, e conquistar clientes. Nosso estudo mostra que, embora as MPMEs catarinenses estejam preocupadas em aumentar o faturamento e reter clientes, ainda é possível melhorar seu desempenho.

Alguns estudos corroboram tal visão, ao indicarem que, para atingir DM, as empresas necessitam desenvolver $\mathrm{CD}$, ser capazes de atrair novos clientes, reter os atuais, aumentar a lucratividade, bem como a participação no mercado, além de oferecer maior qualidade, preços atraentes, e atendimento personalizado (Christensen, 1997; OECD, 2005; Tidd et al., 2005; Tigre, 2006; Chesbrough, 2012; Teece et al., 2014).

Os achados deste estudo se aproximam dos de Braojos-Gomez et al. (2015), ao sugerir que a capacidade da infraestrutura de TI, a pressão do concorrente social, o gerenciamento de marketing e a gestão da inovação são mecanismos fundamentais, por meio dos quais as pequenas empresas aprendem a desenvolver competência em redes sociais.

A hipótese $\mathrm{H} 3$ - "CD influenciam positivamente o desempenho mercadológico", isto é, CD geram DM, não foi sustentada, diferindo dos achados de Wardaya et al. (2019), que receberam 
suporte empírico. Os resultados desta pesquisa reforçam que os mecanismos fundamentais, através dos quais as MPMEs aprendem a desenvolver competências em redes sociais, precisam se desenvolver nessas empresas (Braojos-Gomez et al., 2015). Os resultados também apontaram que a capacidade da infraestrutura de TI, por exemplo, não aumentou nem se reduziu em 2017, a pressão do concorrente social aumentou pouco, e o gerenciamento de marketing e a gestão da inovação aumentaram levemente, o que sugere que as empresas necessitam de um comprometimento interno e investimento em marketing de mídia social para melhorar seu desempenho.

Os resultados também se aproximam da afirmação de Teece et al. (2014), de que a criação de riqueza em regimes de alta mudança tecnológica depende, em grande medida, da capacidade tecnológica, organizacional, e de processos gerenciais da empresa. Ainda, as empresas estudadas têm dificuldade em transformar os dados das MS em conhecimentos pessoais valiosos, e explorar esses insights para criar valor agregado para os clientes (Wieneke \& Lehrer, 2016). Assim, entendese que a validade estatística da hipótese não se confirma, pois as empresas estudadas necessitam fomentar suas CD.

A teoria mostra que as $\mathrm{CD}$ possibilitam desenvolver diferenciais de mercado frente à concorrência (Eisenhardt \& Martin, 2014). Os resultados deste estudo indicam que as MPMEs de Santa Catarina buscam tais diferenciais, mas ainda há espaço para melhorar. Isso se torna visível ao analisarmos a média de pontuação da variável "trabalho para que os recursos de MS não sejam identificados/imitados", bem como a da variável "com o uso de MS, a pressão do concorrente". Portando, as empresas parecem não utilizar ou desconhecem as CD como recurso para melhorar seu DM (Eisenhardt, \& Martin, 2014). Ainda, a relação das CD com a vantagem competitiva está fundamentada na possibilidade de a empresa criar, configurar, integrar e combinar seus recursos (Eisenhardt, \& Martin, 2014), e isso depende da maturidade da organização, pois impacta o DM (Barra \& Ladeira, 2017; Barney \& Hesterly, 2007).

Por fim, Barra e Ladeira (2017) e Barney e Hesterly (2007) afirmam que estratégias diferenciadas (capacidades e recursos raros, inimitáveis, de valor, e organizados) podem gerar valor ao cliente, e gerar desempenho superior. Tal afirmativa colabora para o entendimento de que as MPMEs estudadas necessitam, ainda, absorver e aprender com suas CD.

\section{Conclusão}

O objetivo principal do estudo foi analisar o impacto do uso das mídias sociais nas capacidades dinâmicas e no desempenho mercadológico de micro, pequenas e médias empresas atuantes no estado de Santa Catarina, Brasil. Visando testar a relação entre os constructos, aplicamos um survey para gestores das empresas daquele Estado, obtendo-se 143 respostas. A 
estrutura do estudo integra e sintetiza conceitos e resultados da pesquisa de campo, e os resultados mostram que MS propiciam $\mathrm{CD}$, que MS geram DM, mas que CD não geram DM.

As capacidades das MS parecem ser mais importantes para as pequenas empresas do que para as grandes. Contudo, devido às dificuldades de recursos financeiros, o modelo demonstra que as MPMEs necessitam desenvolver mais CD, para que a eficácia das MS se concretize em DM. Assim, nossos achados confirmam que as empresas têm dificuldades quanto à capacidade de infraestrutura de TI, quanto à pressão de concorrente social, e quanto às capacidades de marketing e gerenciamento de inovação. Esses são mecanismos fundamentais, mediante os quais as empresas aprendem a desenvolver uma competência em redes sociais (Braojos-Gomez et al., 2015).

O estudo também mostra que a competência das MS é mais importante para as empresas pequenas. Entende-se que todos os mecanismos citados (a capacidade da infraestrutura de TI, a pressão social dos concorrentes, o gerenciamento de marketing e a gestão da inovação) são antecedentes fundamentais, por meio dos quais as pequenas empresas aprendem a desenvolver uma competência em redes sociais, apesar de não termos testado a ordem de importância desses mecanismos (Braojos-Gomez et al., 2015).

O estudo demonstrou que as MPMEs necessitam melhorar suas $\mathrm{CD}$, isto é, buscar absorver as informações do mundo digital e transformar os dados das MS em conhecimentos pessoais e valiosos, a fim de explorá-los para criar valor agregado para os clientes. Também contribuiu ao indicar que a criação de riqueza em regimes de alta mudança tecnológica depende, em grande medida, da capacidade tecnológica, organizacional e de processos gerenciais da empresa.

Neste contexto, já foi constatado que para a melhoria do desempenho são cruciais as capacidades de marketing e de orientação para o mercado (Wardaya et al., 2019); entretanto, os resultados desta pesquisa não dão suporte à influência da mediação das capacidades dinâmicas na relação entre o uso das mídias sociais e o desempenho mercadológico. De nada adianta ter presença nas redes sem efetivamente adotar estratégias planejadas de marketing digital.

Conclui-se que as MPMEs do Estado de Santa Catarina estão em um processo de amadurecimento da utilização de mídias sociais para gerar capacidades dinâmicas e desempenho mercadológico. Apresentam avanços, contudo ainda podem melhorar, desenvolvendo capacidades dinâmicas que possibilitem melhor desempenho. É justamente na geração de desempenho, a partir das capacidades dinâmicas, que parecem residir os maiores desafios, pois são os gestores, com suas decisões, os responsáveis pelo desenvolvimento interno e conquista de mercado.

\section{Limitações da pesquisa e sugestões de estudos futuros}

Embora o modelo proposto explique questões referentes às MPMEs do Estado de Santa Catarina, pode haver fatores métricos nas MS dessas empresas, ou percepções dos seus 
stakeholders, que influenciam as CD e o DM das MS. Ainda é preciso considerar que este estudo concentrou-se em percepções dos gestores das empresas, havendo, portanto, motivação para que pesquisadores busquem ampliar a coleta de dados com funcionários e clientes, com a possibilidade de utilizar dados secundários, sobre as mídias sociais das empresas pesquisadas. Estudos futuros podem, também, aumentar o número de empresas da amostra. Outra questão refere-se ao fato de o estudo ter abarcado micro, pequenas e médias empresas. Talvez, com uma só categoria, os resultados fossem distintos. Finalmente, consideramos importante realizar pesquisas adicionais para entender como as capacidades das mídias sociais influenciam o desempenho de grandes empresas em suas inovações, acrescentando a capacidade de marketing como um constructo.

\section{Referências}

Ahani A., Rahim, N. Z. A., \& Nilashi M. (2017). Forecasting social CRM adoption in SMEs: A combined SEM-neural network method. Computers in Human Behavior, 75, 560-578. https://doi.org/10.1016/j.chb.2017.05.032

Barney, J. (1991). Firm resources and sustained competitive advantage. Journal of Management, 17(1), 99-120. https://doi.org/10.1177/014920639101700108

Barney, J.B, \& Hesterly, W.S. (2007). Administração Estratégica e Vantagem Competitiva: casos brasileiros. São Paulo: Pearson.

Barra, G. M. J., \& Ladeira, M. B. (2017). Modelo de maturidade para processos de certificação no sistema agroindustrial do café. REGE-Revista de Gestão, 24(2), 134-148. https://doi.org/10.1016/j.rege.2017.03.004

Braganza, A., Brooks, L., Nepelski, D., Ali, M., \& Moro, R. (2017). Resource management in big data initiatives: Processes and dynamic capabilities. Journal of Business Research, 70, 328337. https://doi.org/10.1016/j.jbusres.2016.08.006

Braojos-Gomez, J., Benitez-Amado, J., \& Llorens-Montes, F. J (2015). How do small firms learn to develop a social media competence? International Journal of Information Management, 35(4), 443-458. https://doi.org/10.1016/j.ijinfomgt.2015.04.003

Callado, A. A. C., Callado, A. L. C., \& Andrade, L. P. (2008). Padrões de utilização de indicadores de desempenho não-financeiros: um estudo exploratório nas empresas de Serra Talhada/PE. ABCustos, 3(2), 1-26.

Castells, M. (2003). A galáxia da Internet. Rio de Janeiro: Jorge Zahar.

Chenhall, R. H., \& Moers, F. (2015). The role of innovation in the evolution of management accounting and its integration into management control. Accounting, Organizations and Society, 47, 1-13. https://doi.org/10.1016/j.aos.2015.10.002

Chesbrough, H. (2012). Inovação aberta: Como criar e lucrar com a tecnologia. Porto Alegre: Bookman. 
Christensen, C. M. (1997). The innovator's dilemma: When new technologies cause great firms to fail. Boston, MA: Harvard Business School Press.

Eisenhardt, K. M., \& Martin, J. A. (2000). Dynamic capabilities: what are they? Strategic Management Journal, 21(10/11), 1105-1121. https://doi.org/10.1002/10970266(200010/11)21:10/11<1105::AID-SMJ133>3.0.CO;2-E

Eisenhardt, K. M., \& Martin, J. A. (2014). O que são as capacidades dinâmicas? In D. P. Lacerda, R. Teixeira, J. Antunes, \& Corcini, S. L. H., Neto (Eds.). Estratégia Baseada em Recursos - 15 artigos clássicos para sustentar Vantagens Competitivas (pp. 211-240). Porto Alegre: Bookman.

Erevelles, S., Fukawa, N., \& Swayne, L. (2016). Big Data consumer analytics and the transformation of marketing. Journal of Business Research, 69(2), 897-904. https://doi.org/10.1016/j.jbusres.2015.07.001

Gupta, M., \& George, J. (2016). Toward the development of a big data analytics capability. Information \& Management, 53(8), 1049-1064. https://doi.org/10.1016/j.im.2016.07.004

Hair, J. F., Jr., William, B., Babin, B., \& Anderson, R. E. (2009). Análise multivariada de dados (6a ed.). Porto Alegre: Bookman.

Instituto Brasileiro de Geografia e Estatística [IBGE]. Agência de Notícias. PNAD Contínua TIC 2017: Internet chega a três em cada quatro domicilios do país. Recuperado de https://agenciadenoticias.ibge.gov.br/agencia-sala-de-imprensa/2013-agencia-denoticias/releases/23445-pnad-continua-tic-2017-internet-chega-a-tres-em-cada-quatrodomicilios-do-pais.

Jantunen, A., Tarkiainen, A., Chari, S., \& Oghazi, P. (2018). Dynamic capabilities, operational changes, and performance outcomes in the media industry. Journal of Business Research, 89, 251-257. https://doi.org/10.1016/j.jbusres.2018.01.037

Jenkins, H. (2006). Convergence culture: Where old and new media collide. New York: New York Univ. Press.

Lam, H. K. S., Yeung, A. C. L., \& Cheng, T.C. E. (2016). The impact of firms' social media initiatives on operational efficiency and innovativeness. Journal of Operations Management, 47, 28-43. https://doi.org/10.1016/j.jom.2016.06.001

Li, L., Su, F., Zhang, W., \& Mao, J. (2017). Digital transformation by SME entrepreneurs: A capability perspective. Information Systems Journal, 28(6), 1129-1157. https://doi.org/10.1111/isj.12153

Lin, Y., \& Wu, L. Y. (2014). Exploring the role of dynamic capabilities in firm performance under the resource-based view framework. Journal of Business Research, 67(3), 407-413. https://doi.org/10.1016/j.jbusres.2012.12.019

Liu, Y., Deng, S., Hu, F., \& Chen, X. (2015). The impacts of unique service resources and habit on e-service loyalty in a highly competitive market. Journal of Systems and Information Technology, 17(4), 336-350. https://doi.org/10.1108/JSIT-06-2015-0050 
Lombardi, M. S., Bertoni, C. E., Nozawa, G. I., Bellato, T. \& Grandi. L. A. (2010) A percepção da incerteza e o desempenho operacional da indústria brasileira de 2007 a 2009. Future Studies Research Journal: Trends and Strategies, 2(2), 56-82.

Martynow, A., \& Shafti, N. (2016). Long-term performance of firms: a review and research agenda. Journal of Strategy and Management, 9(4), 429-448. https://doi.org/10.1108/JSMA-02$\underline{2015-0016}$

Mention, A., Barlatier, P-J., \& Josserand, E. (2019). Using social media to leverage and develop dynamic capabilities for innovation. Technological Forecasting and Social Change, 144, 242250. https://doi.org/10.1016/j.techfore.2019.03.003

Nguyen, B., Yu, X., Melewar, T. C., \& Chen, J. (2015). Brand innovation and social media: knowledge acquired from social media, market orientation, and the moderating role of social media strategic capability. Industrial Marketing Management, 51, 11-25. https://doi.org/10.1016/j.indmarman.2015.04.017

Organization for Economic Cooperation and Development [OECD] (2005). Modernizing Government: The way forward. Paris: OECD Publishing.

Peteraf, M. A. (1993). The cornerstones of competitive advantage: a resource-based view. Strategic Management Journal, 14(3), 179-191. https://doi.org/10.1002/smj.4250140303

Pinho, J. C., \& Prange, C. (2016). The effect of social networks and dynamic internationalization capabilities on international performance. Journal of World Business, 51(3), 391-403. https://doi.org/10.1016/j.jwb.2015.08.001

Pogrebnyakov, N. (2017). A cost-based explanation of gradual, regional internationalization of multinationals on social networking sites. Management International Review, 57(1), 37-64. https://doi.org/10.1007/s11575-016-0281-z

Porter, M.E. (1980). Competitive Strategy: Techniques for Analyzing Industries and Competitors. New York: Free Press.

Saavedra F. U., Andreu J. L., \& Criado, J.R. (2016). The impact of social media marketing on the relationship among dynamic capabilities and performance. In C. Campbell, \& J. J. Ma (Eds.). Looking forward, looking back: Drawing on the past to shape the future of marketing. Proceedings of the 2013 World Marketing Congress. Cham: Springer.

Santos, N. M., Bronzo, M., Oliveira, M. P. V., \& Resende, P. T. V. (2014). Cultura organizacional, estrutura organizacional e gestão de pessoas como bases para uma gestão orientada por processos e seus impactos no desempenho organizacional. Brazilian Business Review, 11(3), 106-129. https://doi.org/10.20397/2177-6652/2017.v17i1.915

Serviço Brasileiro de Apoio às Micro e Pequenas Empresas [SEBRAE] (2017). Anuário do trabalho nos pequenos negócios 2015 (8a ed.). São Paulo:DIEESE.

Teece, D. J., Pisano, G., \& Shuen, A. (1997) Dynamic capabilities and strategic managment. Strategic Managment Journal, 18(7), 509-533. https://doi.org/10.1002/(SICI)10970266(199708)18:7<509::AID-SMJ882>3.0.CO;2-Z 
Teece, D., Pisano, G., \& Shuen, A. (2014). Capacidades dinâmicas e gestão estratégica. In D. P. Lacerda, R. Teixeira, J. Antunes, \& Corcini, S. L., Neto (Eds.) Estratégia Baseada em Recursos. Porto Alegre: Bookman.

Telles, A. (2011). A revolução das mídias sociais: Cases, conceitos, dicas e ferramentas. São Paulo: M. Books.

Tidd, J., Bessant, J., \& Pavitt, K. (2005). Managing innovation: Integrating technological, market and organizational change (3 ed.). Chichester: John Wiley \& Sons.

Tigre, P. B. (2006). Gestão da inovação. Rio de Janeiro: Campus.

Venkatraman, N., \& Ramanujam, V. (1986). Measurement of business performance in strategy research: a comparison of approaches. Academy of Management Review, 11(4), 801-814. https://doi.org/10.5465/amr.1986.4283976

Wamba, S. F., Gunasekaran, A., Akter, S., Ren, S. J., Dubey, R., \& Childe, S. J. (2017). Big data analytics and firm performance: Effects of dynamic capabilities. Journal of Business Research, 70, 356-365. https://doi.org/10.1016/j.jbusres.2016.08.009

Wang, C. L., \& Ahmed, P. K. (2007) Dynamic capabilities: a review and research agenda. International Journal of Management Reviews, 9(1), 31-51. https://doi.org/10.1111/j.1468$\underline{2370.2007 .00201 . x}$

Wang, Z., \& Kim, H. G. (2017). Can social media marketing improve customer relationship capabilities and firm performance? Dynamic capability perspective. Journal of Interactive Marketing, 39, 15-26. https://doi.org/10.1016/j.intmar.2017.02.004

Wardaya, A., Idris, S., So, I. G., \& Bandur, A. (2019). Mediating effects of digital marketing on dynamic capability and firm performance: Evidence from small and medium-sized enterprises (SMEs) in Indonesia. International Journal of Recent Technology and Engineering (IJRTE), 8(5C), 692-696. DOI:10.35940/ijeat.E1098.0585C19

Wieneke, A., \& Lehrer, L. (2016). Generating and exploiting customer insights from social media data. Electronic Markets, 26(3), 245-268. doi:10.1007/s12525-016-0226-1

Zavattaro, S. M., \& Daspit, J. J. (2016). A grounded theoretical approach to understanding innovation in destination marketing organizations. Journal of Vacation Marketing, 22(4), 349364. doi:10.1177/1356766715623826

Zollo, M., \& Winter, S. G. (2002). Deliberate learning and the evolution of dynamic capabilities. Organization Science, 13(3), 339-351. https://doi.org/10.1287/orsc.13.3.339.2780. 
Apêndice 1 - Questionário

Assinale o principal setor de atividade da sua empresa:

Comércio

Indústria

Prestação de serviços

Indique o número de funcionários de sua empresa:

Indique a cidade-sede de sua empresa:

Quanto ao uso de cada mídia social por sua empresa no ano de 2017, escolha uma das opções, sendo:

"1=nenhum uso" e "5=máximo uso".

Facebook

Google+

Instagram

LinkedIn

Messenger

Pinterest

Skype

Snapchat

Telegram

Tumblr

Twitter

WhatsApp

YouTube

Outro (especifique):

Nas questões a seguir, posicione o cursor e clique na barra, conforme sua percepção em relação à empresa, sendo $0=$ reduziu $10 \%$ ou mais, $50=$ não reduziu nem aumentou e $100=$ aumentou $10 \%$ ou mais.

Para responder, favor considerar o ano de 2017:

A atuação da minha empresa nas mídias sociais consistiu em:

Utilização das informações advindas das mídias sociais para conhecer meu cliente:

Monitoramento das mídias sociais da nossa empresa:

Planejamento das estratégias para as mídias sociais da nossa empresa:

Interação de nossa empresa com o consumidor, nas mídias sociais:

Ações realizadas por nossa empresa a partir dos resultados das mídias sociais:

Investimento de nossa empresa em mídias sociais:

Divulgação dos meus produtos ou serviços nas mídias sociais:

Trabalho para garantir que nossos recursos de mídias sociais não fossem facilmente identificados ou imitados pelos concorrentes:

Compartilhamento das informações das mídias sociais entre os setores da nossa empresa, visando uma gestão integrada:

Com o uso das mídias sociais, a reorganização ou construção da equipe de negócios ou das competências internas da empresa:

Com o uso das mídias sociais, a capacidade da infraestrutura de TI de nossa empresa:

Com o uso das mídias sociais, a pressão do nosso concorrente:

Com o uso das mídias sociais, os esforços para o gerenciamento de marketing da nossa empresa:

Com o uso das mídias sociais, nossos esforços para a gestão da inovação:

Com o uso das mídias sociais, nossa agilidade de resposta às mudanças do ambiente competitivo:

O nível de satisfação dos nossos clientes:

O nível de retenção dos nossos clientes:

O número de novos clientes:

O faturamento da nossa empresa:

O volume de vendas da nossa empresa: 



\title{
$\neg$ HE EFFECT OF THE USE OF SOCIAL MEDIA AND DYNAMIC CAPABILITIES ON MARKET PERFORMANCE OF MICRO, SMALL AND MEDIUM-SIZED FIRMS
}

\author{
${ }^{1}$ Silvia Spagnol Simi dos Santos \\ ${ }^{2}$ Sérgio Begnini \\ ${ }^{3}$ Carlos Eduardo Carvalho
}

Objective: This article analyzes the impact of the relationship between the use of social media, dynamic capabilities, and market performance on companies, as well as the mediating role of dynamic capabilities in the relationship between the use of social media and market performance.

Method: We adopted a quantitative and descriptive methodology, and collected data through a survey, with a sample of 143 companies. We carried out factor analysis by using the SPSS ${ }^{\circledR}$ software, and structural equation modeling by using the AMOS® software.

Originality/Relevance: This study deepens the understanding of the role of social media in turbulent environments, and its relationship with dynamic capabilities and market performance in micro, small and medium-sized companies.

Results: The hypotheses were tested and showed a positive effect of the use of social media on dynamic capabilities, as well as of the use of social media on market performance. However, dynamic capabilities did not mediate the relationship between the use of social media and market performance.

Theoretical/methodological contributions: The results contribute to science by highlighting the role of social media in turbulent environments, and its relationship with dynamic capabilities and market performance in micro, small and medium-sized companies. They also contribute theoretically and empirically by identifying factors that allow maximizing organizations' productivity.

Social/management contributions: The biggest challenge for managers of micro, small and mediumsized firms is to turn social media data into valuable personal insights, since exploiting these insights to add value is the biggest barrier for these companies.

Keywords: Market performance. Dynamic capabilities. Social media. Micro, small and medium-sized enterprises.

\section{How to cite the article:}

Santos, S. S. S. dos, Begnini, S., \& Carvalho, C. E. (2020). The effect of the use of social media and dynamic capabilities on market performance of micro, small and medium-sized firms. Brazilian Journal of Marketing, 19(1), 164-185. https://doi.org/10.5585/remark.v19i1.17346. 


\section{Introduction}

The purpose of this study was to examine the Dynamic Capabilities (DC) approach in strategic management, focused on the processes related to the use of Social Media (SM), regarding firms' Market Performance (MP). Social media are websites created on the internet that allow collaborative creation of content, its sharing through different information formats, and social interaction (Telles, 2011), which means that a company present on social media can create relationships and make conversations.

In dynamic environments (Eisenhardt \& Martin, 2000) such as social media, where it is possible to acquire and generate resources in real time, DC consist of strategic decision-making processes, which manipulate these resources to get value. Teece, Pisano and Shuen $(1997,2014)$ claim that DC are essential, especially in rapidly changing environments, and this varies according to market dynamism (Eisenhardt \& Martin, 2000). Clearly, companies that operate in dynamic business environments constantly face the challenge of adapting to new conditions, through different ways of combining dynamic capabilities with internal changes that lead them to a high performance of their business models (Jantunen, Tarkiainen, Chari, \& Oghazi, 2018).

Micro, small, and medium-sized enterprises (MSMEs) stand out in the Brazilian scenario for their contribution to job creation and to the increase of country's wealth, substantially helping the national economy. In 2015, they were more than 6.8 million firms (Sebrae, 2017). Internet has allowed firms to get precise information on their customers, markets, and competitors (Wardaya, Idris, So, \& Bandur, 2019); in short, on all their stakeholders, and currently it is essential for marketing.

Brazil is among the countries with the largest number of social media users in the world, with an active population of 126.3 million users, and internet reached $74.9 \%$ of Brazilian homes in 2017 (IBGE, 2019). Companies have increasingly used social media as new ways to connect, communicate, and engage with a wide range of stakeholders (Mention, Barlatier, \& Josserand, 2019). In this business set, immersion in SM becomes a differential, especially for those that wish to improve their market performance, since it brings dynamism to the corporate environment. As the strategies and tactics that social media offers are countless, we present the central question of this research: What is the relationship between social media, dynamic capabilities, and market performance in micro, small and medium enterprises? Thus, the objective was to identify the impact of the relationship between the use of social media, dynamic capabilities, and market performance, as well as the mediating role of dynamic capabilities on the relationship between the use of social media and market performance.

We identified several studies similar to this research, such as the one by Wamba et al. (2017), which confirmed direct and indirect impacts of the use of big data tools on the performance 
of firms, where dynamic capabilities played a mediating role in improving knowledge, thus, performance. Wang and Kim (2017) investigated the use of social media from the perspective of dynamic organizational capabilities. Saavedra, Andreu and Criado (2016) found that companies should use online social networks intensively, to enhance the relationship between market orientation and performance. Wardaya et al. (2019) tested the effect of dynamic capabilities on firms' performance and digital marketing capacity, as well as the role of digital marketing.

We tested such arguments through primary data collected in 2017, from a sample of 143 companies. We did the survey on the internet, with a form sent by e-mail to the managers of these firms. To test the results, we used structural equation modeling. The results provided evidence of a positive effect between the use of SM and MP, and between DC and MP. However, DC did not mediate the relationship between SM and MP.

The article has five sections. Section 2 presents the literature review on SM, DC and MP, besides hypotheses' building. Section 3 shows the methodological procedures, and section 4 describes the analysis and discussion of results. Section 5 presents the conclusion, followed by the limitations and suggestions for future studies, in section 6.

\section{Literature review and hypotheses}

Dynamic Capabilities are the strategies developed by a company, through which managers access resources, either by acquisition or by creation, and use them, generating new strategies and adding value to the organization. This allows them to develop market differentials in the face of competition, and gives managers greater possibilities to integrate, build and reconfigure both the internal and external competencies of the firm. Hence, dynamic capabilities are sources of sustainable competitive advantage for organizations (Eisenhardt \& Martin, 2014).

DC enable the company that operates in complex and fast changing environments to detect, analyze and understand the sources and procedures that involve wealth creation and appropriation (Teece et al., 2014). The study of DC helps to comprehend how companies act to reconfigure their resources, in an environment of uncertainty, or even in peaceful ones, in order to achieve high market performance. Thus, the relationship between DC and competitive advantage stands on the possibility that the company creates, configures, integrates, and combines its resources (Zollo \& Winter, 2002; Wang \& Ahmed, 2007; Eisenhardt \& Martin, 2014).

DC show that the competence of companies to change and reorganize new resources, under different configurations, is more relevant than the resources themselves, for the creation of sustainable competitive advantage (Teece et al., 2014); therefore, DC also change organizations' resource base through organizational strategies. The authors highlight that business strategy 
provides a competitive advantage, supported by the company's performance through the several organizational routines done by employees; as the number of these routines increases, it is possible to develop organizational skills. Organizational competence exists when people become aware of the result of their actions in the firm's overall performance, through the resources they use (Zollo \& Winter, 2002).

Due to the set of available resources and their reconfiguration, firms can achieve positive results. Whether based on strategic positioning (Porter, 1980), or on the resource-based view (RBV) (Barney, 1991; Peteraf, 1993), or even with a focus on DC (Teece et al., 1997; Eisenhardt \& Martin, 2000), companies' market performance is a subject of research and debate. The search for positive results is continuous in all companies, since sustainability in the market depends on the accomplished performance.

Regarding the use of social media by companies, in order to achieve performance in their market, dynamic capabilities are driving forces in the process, as they allow companies, in a growing market, to identify important signs, evaluate new processes, services or products, and respond to changes in that market (Wardaya et al., 2019). Social media has changed the way a company connects, leading to different possibilities in different organizational functions, from marketing to operations management, and to the development of new products (Lam, Yeung, \& Cheng, 2016). Therefore, the immersion of companies in SM can change the way they relate to their stakeholders. This is because social networks provide a platform for sharing experiences, accumulating knowledge and organizational learning (Nguyen, Yu, Melewar, \& Chen, 2015).

\subsection{Use of social media tools, dynamic capabilities, and market performance in micro, small and medium-sized firms}

Contemporary research has studied the use of social media, market performance and dynamic capabilities; however, literature is scarce regarding the relationship between these variables. Gupta and George (2016) identified several resources that, combined, build Big Data Analytics capacity, and created an instrument to measure such capacity and the company's performance. Lam et al. (2016) examined the impact of social media initiatives on companies' operational efficiency and innovation capacity. Wang and Kim (2017) examined how the use of social media can help companies create new management capacities in consumer relationship, in their quest to improve business performance. Wamba et al. (2017) studied the effects of analytics capacity of big data on company performance, as well as the mediating effects of dynamic capabilities on the relationship between big data analytics capacity and company performance. Ahani, Rahim and Nilashi (2017) sought the predictive elements of the adoption of a strategy of relationship management with customers in small and medium-sized companies. 
Another study investigated how MSMEs entrepreneurs with small capacities and limited resources changed cross-border e-commerce in China ( $\mathrm{Li}, \mathrm{Su}$, Zhang, \& Mao, 2017). The study revealed key stages in the process, including "renewing managerial cognition, developing managerial social networks, building a business team, and creating organizational capacity" (Li et al., 2017, p. 21).

Braojos-Gomez, Benitez-Amado and Llorens-Montes (2015) examined the critical mechanisms by which small businesses learn to develop competence in social networks, and concluded that they use their IT infrastructure capacity, the pressure of the social competitors, marketing management, and innovation management. Technological capability, the capacity of management processes, and organizational capacity are directly related to the firm's technological change (Teece et al., 2014), and this relationship has an impact on wealth creation. Within environments of increasing and frequent changes, mainly involving social media, MSMEs need to achieve and keep market performance. One possibility is to develop dynamic capabilities through social media, in order to design and implement the best business strategy or practice. DC exploit the company's internal and external competencies to face the turbulent and changing environment. With that in mind, we developed the first hypothesis:

\section{Hypothesis 1: The use of social media positively affects MSMEs' dynamic capabilities.}

Studies on social media and dynamic capabilities comprise a number of elements relevant to the areas of business and marketing. Zavattaro and Daspit (2016) addressed issues related to innovation, while Pogrebnyakov (2017) and Pinho and Prange (2016) focused on aspects of companies' internationalization. Braganza, Brooks, Nepelski, Ali and Moro (2017) addressesd management; Braojos-Gomez et al. (2015) and Wieneke and Lehrer (2016) focused on the return on assets; Liu, Deng, Hu and Chen (2015) and Erevelles, Fukawa and Swayne (2016) addressed marketing and market orientation. The study by Wieneke and Lehrer (2016) listed the capabilities required from companies to change SM data into valuable knowledge, and explore it to add value to customers. Wang and Kim (2017) explored the use of SM in B2B marketing, from a perspective of dynamic organizational capacity.

Lam et al. (2016) found social media initiatives as positive impacts on operational efficiency and innovation. Thus, the use of SM can become a decisive source of market performance, since we already live in a culture of convergence (Jenkins, 2006). This culture changes the relationship of companies with suppliers, employees and buyers, and affects production processes, the relationship with other companies, and the evaluation by society (Castells, 2003), and, in a stronger way, the consumer market. On the other hand, the improper use of SM also has negative results on companies' performance. Based on these arguments, we developed the second research hypothesis: 
Hypothesis 2: The use of social media positively affects MSMEs' market performance

\subsection{Influence of dynamic capabilities on market performance}

Faced with competition, a complex environment, and market uncertainties, companies need elements that enable them to gain a sustained competitive advantage, thus achieving high performance (Martynow \& Shafti, 2016). One of the factors that directly affect market performance is the maturity of the organization, which is a result of its path and of constant reviews and improvements of its internal processes (Barra \& Ladeira, 2017). With the implementation of different strategies, based on rare, inimitable, valuable, and organized capacities and resources, the company will be able to create value for the customer and achieve superior performance in the market (Barney \& Hesterly, 2007).

It is possible to identify and measure companies' MP by using some data and information. Over time, authors have identified and used different variables: customer satisfaction, employee satisfaction, profitability, sales growth, and company reputation (Venkatraman \& Ramanujam, 1986). Callado, Callado and Andrade (2008) referred to production capacity, increase in the number of customers, customer loyalty, investment in training, advertising and modernization, product/service quality, brand value, and sales volume. Lombardi, Bertoni, Nozawa, Bellato, \& Grandi (2010) addressed sales volume and decision-making; and Santos, Bronzo, Oliveira, \& Resende (2014) identified customer loyalty, attraction of new customers, sales volume, market share, and customer satisfaction.

In a previous study, Wardaya et al. (2019) tested the influence of dynamic capabilities on the performance of Indonesian firms, with positive results. Dynamic capabilities increase the speed and efficiency of small and medium-sized companies' responses to environmental turbulence, which in turn improves performance. In order to achieve a favorable MP, companies need to develop DC, that is, learn to reinvent themselves, constantly. They must be able to attract new customers, retain current ones, increase profitability, as well as market share, in addition to offering higher quality, attractive prices and customized service (Christensen, 1997; OECD, 2005; Tidd, Bessant, \& Pavitt, 2005; Tigre, 2006; Chesbrough, 2012). By developing DC, companies will be better prepared to compete and operate in the market, and achieve a better performance. Thus, we developed the third research hypothesis:

Hypothesis 3: Dynamic capabilities positively affect MSMEs' market performance 


\subsection{The effect of dynamic capabilities}

We found few studies on the mediating effect of dynamic capabilities on the relationship between the use of social media and market performance. Saavedra et al. (2016) concluded that companies should use online social networks intensively to enhance the relationship between market orientation and performance. If companies actively participate in online social networks and really have a strategy for social media marketing, investing heavily in their use, in addition to a manager or a digital marketing agency, they can take advantage of the potential of these networks as a marketing tool. The results show that it is not sufficient to have some profiles and carry out some basic actions of social media marketing, but rather put a strong and dedicated effort on this type of marketing, in order to observe a real impact on performance.

The study by Wamba et al. (2017) is similar to ours, and showed a relationship between the use of tools related to big data capabilities and performance, and also between those and firms' dynamic capabilities; moreover, it showed that DC mediate the relationship between big data capacity and performance. Although it addressed the use of information created by social media, through big data, and our study focuses specifically on the use of social media, its findings can contribute to our investigation, mainly because they confirm direct and indirect impacts of big data on firms' performance, and that dynamic capabilities play a mediating role for increasing knowledge, thus, performance.

Wardaya et al. (2019) studied managers of small and medium-sized companies in Indonesia, using a model similar to ours, and tested the influence of dynamic capabilities on companies' performance and digital marketing capacity, as well as the reverse, that is, the influence of digital marketing capacity on firms' performance. They also tested the mediating effect of digital marketing on this relationship, with positive results.

Literature is scarce on the mediating effect of Dynamic Capabilities on the relationship between the use of social media by companies and their market performance, thus it is worth examining this issue. We then developed the fourth hypothesis:

H4: Dynamic capabilities mediate the relationship between the use of social media tools and MSMEs' market performance.

Figure 1 presents the conceptual model of the research, showing the constructs' mutual influence. 
Figura 1 - Conceptual model

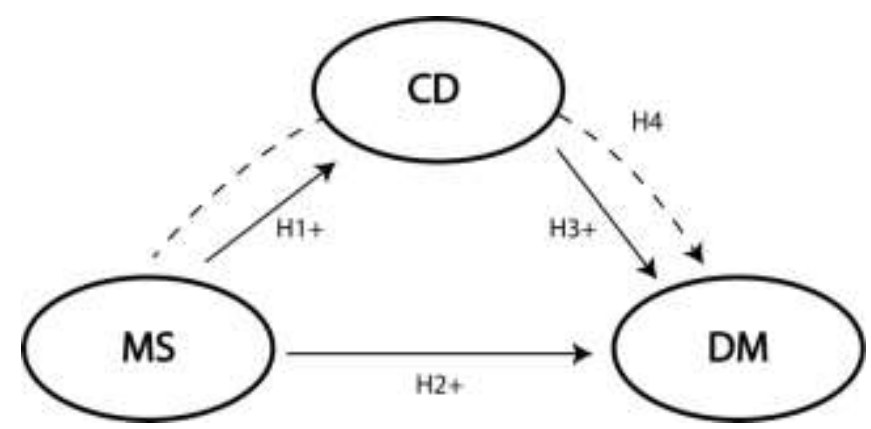

Source: The authors, 2018.

\section{Method}

The main objective of this research was to understand the relationship between social media, dynamic capabilities, and market performance. Our focus was on micro, small and medium-sized companies in industry, commerce, and services that operate in the state of Santa Catarina. We got primary data through a survey, by sending a questionnaire to companies' managers. We tried to gather the highest possible number of companies, in order to improve our contribution to the literature.

\subsection{Data and sample}

We carried out the study with micro, small and medium-sized companies that are part of the Federation of Business Associations of Santa Catarina, Brazil, which assembles more than 35 thousand companies affiliated to 148 Business Associations in the State of Santa Catarina. We characterized the sample according to the Brazilian Service for the Support of Micro and Small Enterprises (Sebrae), which uses the size of companies to rank them. We received 172 responses, of which 143 became the study sample, as they were complete and met the research criteria (micro, small and medium-sized companies). The research was descriptive and quantitative, since it sought to measure the effect of the use of social media on dynamic capabilities, and its relationship with market performance.

\subsection{Instrument's preparation and validation}

To build and validate the research instrument, we first created the assertions based on the literature. It consists of primary data, collected by means of a questionnaire with 26 questions, applied through the SurveyMonkey platform. Four questions addressed companies' identification, and one referred to understanding the use of social media, structured on a 5-point Likert scale, where 
1 meant "no use" and 5 "maximum use". Other 21 statements, shown in Table 1, aimed to get a position (score) from the respondents, on a continuous scale from 0 to 100 , where 0 meant "reduced $10 \%$ or more", 50 indicated "neither reduced nor increased" and 100 meant "increased $10 \%$ or more". Figure 2 shows the outline of one of the assertions in the questionnaire, and this is how they were all arranged. The full questionnaire is available in Appendix 1.

Figure 2 - Draft of the assertions

monitoramento das midias sociais da nossa empresa:

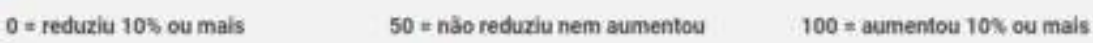

(monitoring our firm's social media; $0=$ reduced $10 \%$ or more; $50=$ neither reduced nor increased; $100=$ increased $10 \%$ or more)

Source: The authors, 2018.

Table 1 shows the latent variables, the assertions of each latent variable, and the authors that created them. We conducted a pre-test of the resulting instrument with three professionals, and a specialist in the field validated it. After validation, we sent the questionnaire by email to the research population. These variables establish important indicators, as they allow to compare and measure companies' performance. The questionnaire had four parts: the first characterized the respondent; the second sought to identify the use of SM by companies; the third addressed the DC of the companies; and the fourth tried to measure the MP of each firm.

Table 1 - Authors that formulated the assertions of each construct

\begin{tabular}{|c|c|c|}
\hline Authors & Assertions & Construct \\
\hline $\begin{array}{l}\text { Nguyen et al. }(2015) \\
\text { Braojos-Gomez et al. } \\
(2015) \\
\text { Wieneke \& Lehrer } \\
(2016) \\
\text { Lam et al. (2016) }\end{array}$ & $\begin{array}{l}\text { 1. Company's action on SM } \\
\text { 2. Use of SM information to know the customer } \\
\text { 3. SM monitoring } \\
\text { 4. Company's strategy planning for SM } \\
\text { 5. Interaction with the consumer through SM } \\
\text { 6. Company's actions from SM results } \\
\text { 7. Company's investment in SM } \\
\text { 8. Promotion of products/services on SM }\end{array}$ & SM \\
\hline $\begin{array}{l}\text { Teece et al. (1997) } \\
\text { Eisenhardt \& Martin } \\
(2000) \\
\text { Zollo \& Winter (2002) } \\
\text { Wang \& Ahmed (2007) } \\
\text { Lin \& Wu (2014) } \\
\text { Teece et al. } \text { (2014) } \\
\text { Eisenhardt (2014) } \\
\text { Braganza } \text { et al. (2017) }\end{array}$ & $\begin{array}{l}\text { 1. Work to prevent SM resources from being identified/imitated } \\
\text { 2. Sharing information on SM with company's sectors, for an } \\
\text { integrated view. } \\
\text { 3. With the use of SM, reorganization of the business team or internal } \\
\text { competences } \\
\text { 4. With the use of SM, enhancement of IT infrastructure } \\
\text { 5. With the use of SM, pressure by competitors } \\
\text { 6. With the use of SM, efforts for MKT management } \\
\text { 7. With the use of SM, efforts for innovation management } \\
\text { 8. With the use of SM, fast responsiveness to changes in the } \\
\text { competitive environment }\end{array}$ & DC \\
\hline
\end{tabular}




\begin{tabular}{|c|c|c|}
\hline $\begin{array}{l}\text { Venkatraman \& } \\
\text { Ramanujam (1986) } \\
\text { Tidd et al. }(2005) \\
\text { Barney \& Hesterly } \\
\text { (2007) } \\
\text { Callado et al. } \text { (2008) } \\
\text { Lombardi } \text { et al. }(2010) \\
\text { Santos et al. } \text { (2014) } \\
\text { Liu et al. } \text { (2015) }\end{array}$ & $\begin{array}{l}\text { 1. Level of customer satisfaction } \\
\text { 2. Level of customer retention } \\
\text { 3. Number of new customers } \\
\text { 4. Company income } \\
\text { 5. Company's sales volume }\end{array}$ & MP \\
\hline
\end{tabular}

Source: Research data, 2018.

After data collection, we used the Kolmogorov-Smirnov and Shapiro-Wilk tests to check for normality. Next, we used exploratory factor analysis (EFA) to assess the dimensionality of the scales, using the SPSS ${ }^{\circledR}$ software, and carried out structural equation modeling (SEM), using the AMOS® software. We also used the PROCESS V3.4 Andrew F. Hayes macro to check the existence of mediation between variables. As control variables, we tested the size of the companies and their sector. For such, we conducted regression analyses and analysis of variance (ANOVA), considering the control variables as independent; we found no influence of them on the constructs.

\section{Results}

In 2015, micro, small and medium-sized firms in Brazil comprised a population above 6.8 million establishments (Sebrae, 2017). In this business set, immersion in SM becomes a differential, especially for those that wish to improve their market performance. To facilitate data analysis of MSMEs that participated in the research, we first carried out a descriptive analysis, followed by SEM, to test the formulated hypotheses.

\subsection{Data descriptive analysis}

In the sample (services, commerce, and industry), the service sector has the largest number of companies, followed by commerce and industry. In terms of performance, micro-firms stand out, in the three sectors, and account for $65.03 \%$, while small ones are $29.37 \%$, and medium-sized are $5.60 \%$. By sector, industry, with $52.67 \%$, has the largest number of employees, followed by services, with $31.62 \%$, and commerce, with $15.71 \%$. It became evident that companies use social media in some way. Based on the answers, Table 2 shows the average usage of each social media, by sector, and the overall mean. 
Table 2 - Average use of Social Media, by sector, and the overall mean

\begin{tabular}{|c|c|c|c|c|c|c|c|c|c|c|c|c|c|}
\hline 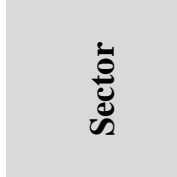 & 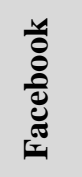 & $\begin{array}{l}\frac{+}{0} \\
\stackrel{0}{0} \\
\stackrel{0}{0}\end{array}$ & 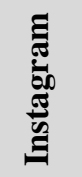 & 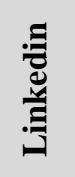 & 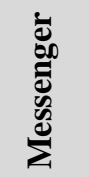 & & 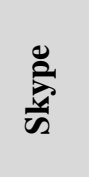 & 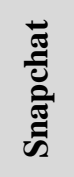 & 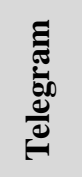 & $\stackrel{\grave{\bar{E}}}{\underline{\Xi}}$ & 氖 & 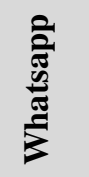 & 晜 \\
\hline $\mathrm{m}$ & & 11 & & 1 & 2,4 & 11 & 2.1 & & & 1.0 & 1.5 & 43 & 1.79 \\
\hline & 3.16 & 1.89 & 1.95 & 2.4 & 1,8 & 10 & 3.0 & 1.0 & 1. & 1.00 & 1.2 & 3.9 & 1.79 \\
\hline Services & 3.77 & 2.21 & 2.62 & 1.97 & 2,28 & 1.31 & 2.57 & 1.2 & 1.1 & 1.01 & 1.47 & 4.38 & 2.00 \\
\hline Overall & 3.65 & 2.07 & 2.48 & 1.96 & 2,19 & 1.14 & 2.57 & 1.15 & 1.08 & 1.03 & 1.39 & 4.24 & 1.86 \\
\hline
\end{tabular}

Source: Research data, 2018.

Respondents selected, between 1 and 5, the answer that best represented their reality, where 1 meant "no use of that social media" and 5 "maximum use of that social media". The overall mean for the use of each one shows that the largest use is of Whatsapp, followed by Facebook, and the least use is of Tumblr, Telegram, Pinterest, and Snapchat. Besides analyzing the use of SM, we also identified some aspects related to each of the three research constructs.

The construct "Social Media" [SM] related to eight assertions, whose results indicated the mean of the answers above 50 points, showing that MSMEs in Santa Catarina increased the use of social media in 2017, compared to the previous year. The largest growth refers to the dissemination of products or services on SM, which got the highest average (74.68). The smallest growth refers to the work to prevent SM resources from easy identification or imitation by competitors, which averaged 53.14.

The construct "Dynamic Capabilities" [DC] covered eight assertions, and reached a mean above 50 points. This shows that companies somehow sought to develop or reconfigure internal and external competencies to adapt to changes (Teece et al., 1997), and achieve competitive advantage (Lin \& Wu, 2014). The agility to respond to changes in the competitive environment, by using social media, was the variable with the highest average (70.66). The lowest (51.13) regards the use of social media for enhancing IT infrastructure.

The third construct, "Market Performance" [MP], comprised five statements and, on average, indicates that MSMEs were able to achieve positive results. Understanding MP is important for helping these firms to identify threats and opportunities, to keep attention to market changes (Chenhall \& Moers, 2015), and to view opportunities for business innovation. Compared to 2016, the highest growth was on the level of customer satisfaction, with average of 70.73 , and the lowest growth was on the level of customer retention, with average of 63.10 . Table 3 shows the descriptive values for each indicator. 
Table 3 - Variables and description of research data

\begin{tabular}{|c|c|c|c|c|c|c|}
\hline Construct & Variables & $\mathbf{M}$ & SD & $\begin{array}{l}\text { Variati } \\
\text { on }\end{array}$ & $\underset{\mathbf{y}}{\text { Assimetr }}$ & $\begin{array}{l}\text { Kurtos } \\
\text { is }\end{array}$ \\
\hline \multirow{8}{*}{$\begin{array}{l}\text { Social } \\
\text { Media } \\
\text { (SM) }\end{array}$} & Company's action on SM & 72,31 & 26,31 & 692,39 &,- 871 & ,268 \\
\hline & Use of SM information to know the customer & 66,65 & 24,04 & 577,93 &,- 188 &,- 515 \\
\hline & SM monitoring & 69,83 & 26,08 & 679,92 &,- 709 & , 195 \\
\hline & Company's strategy planning for SM & 69,06 & 25,54 & 652,23 &,- 605 & ,103 \\
\hline & Interaction with the consumer through SM & 74,07 & 25,18 & 634,02 &,- 838 & ,369 \\
\hline & Company's actions from SM results & 64,91 & 24,91 & 620,51 &,- 358 &,- 138 \\
\hline & Company's investment in SM & 62,49 & 27,17 & 738,24 &,- 352 &,- 184 \\
\hline & Promotion of products/services on SM & 74,68 & 25,66 & 658,60 &,- 930 & 407 \\
\hline \multirow{8}{*}{$\begin{array}{l}\text { Dynamic } \\
\text { Capabiliti } \\
\text { es (DC) }\end{array}$} & $\begin{array}{l}\text { Work to prevent SM resources from being } \\
\text { identified/imitated }\end{array}$ & 53,14 & 25,26 & 638,16 &,- 012 & ,242 \\
\hline & $\begin{array}{l}\text { Sharing information on SM with company's } \\
\text { sectors, for an integrated view }\end{array}$ & 59,19 & 28,49 & 811,59 &,- 281 &,- 341 \\
\hline & $\begin{array}{l}\text { With the use of SM, reorganization of the } \\
\text { business team or internal competences }\end{array}$ & 57,52 & 24,61 & 605,50 &,- 177 & ,423 \\
\hline & $\begin{array}{l}\text { With the use of SM, enhancement of IT } \\
\text { infrastructure }\end{array}$ & 51,13 & 23,25 & 540,34 &,- 217 & 966 \\
\hline & With the use of SM, pressure by competitors & 58,57 & 22,71 & 515,87 &,- 120 & ,731 \\
\hline & $\begin{array}{l}\text { With the use of SM, efforts for MKT } \\
\text { management }\end{array}$ & 65,89 & 27,10 & 734,58 &,- 657 & 204 \\
\hline & $\begin{array}{l}\text { With the use of SM, efforts for innovation } \\
\text { management }\end{array}$ & 67,10 & 24,99 & 624,70 &,- 472 &, 152 \\
\hline & $\begin{array}{l}\text { With the use of SM, fast responsiveness to } \\
\text { changes in the competitive environment }\end{array}$ & 70,66 & 24,66 & 608,25 &,- 646 & ,201 \\
\hline \multirow{5}{*}{$\begin{array}{l}\text { Market } \\
\text { Performa } \\
\text { nce (MP) }\end{array}$} & Level of customer satisfaction & 70,73 & 21,45 & 460,07 &,- 612 & ,679 \\
\hline & Level of customer retention & 63,10 & 24,09 & 580,54 &,- 366 & ,272 \\
\hline & Number of new customers & 67,87 & 25,41 & 645,46 &,- 695 & ,466 \\
\hline & Company income & 63,64 & 24,33 & 592,02 &,- 281 & ,334 \\
\hline & Company's sales volume & 65,12 & 24,32 & 591,53 &,- 442 & ,415 \\
\hline
\end{tabular}

Source: Research data, 2018.

\subsection{Exploratory data analysis}

As data approach normality, with relatively low kurtosis and asymmetry values, before proceeding to hypothesis testing through Structural Equation Modeling (SEM), we carried out an exploratory factor analysis (EFA) to evaluate the scales' dimensionality. Studies with reflective constructs use EFA, by gathering the variables into an ideal number of factors, to better represent the data (Hair, William, Babin, \& Anderson, 2009). We used the method of main components and the eigenvalue criterion equal to 1 (one), which allowed extracting the factors.

For the correct adequacy of the method, variables "Company's strategy planning for SM" and "With the use of SM, fast responsiveness to changes in the competitive environment" were excluded. The degree of explanation of the Kaiser-Meyer-Olkin test (KMO) was 0.894, and the Chi-square value of the Bartlett test was 1813.08, significant at 0.000 , which indicated data 
adequacy (Hair et al., 2009). The explained variance was $67.05 \%$, meeting the values indicated by the literature.

Next, we carried out the reliability test, using Cronbach's Alpha for the three constructs. Cronbach's alpha values for the SM, DC and MP constructs were 0.912, 0.890, and 0.893, respectively. Thus, as recommended by Hair et al. (2009), the model's internal consistency and reliability measures were adequate to proceed with Structural Equation Modeling.

\subsection{Structural equation modeling}

Through the exploratory analysis, we reached a more adjusted model, composed of seven indicators for the construct DC, seven for SM, and five for MP, as shown in Figure 3.

Figure 3 - Variables and description of research data

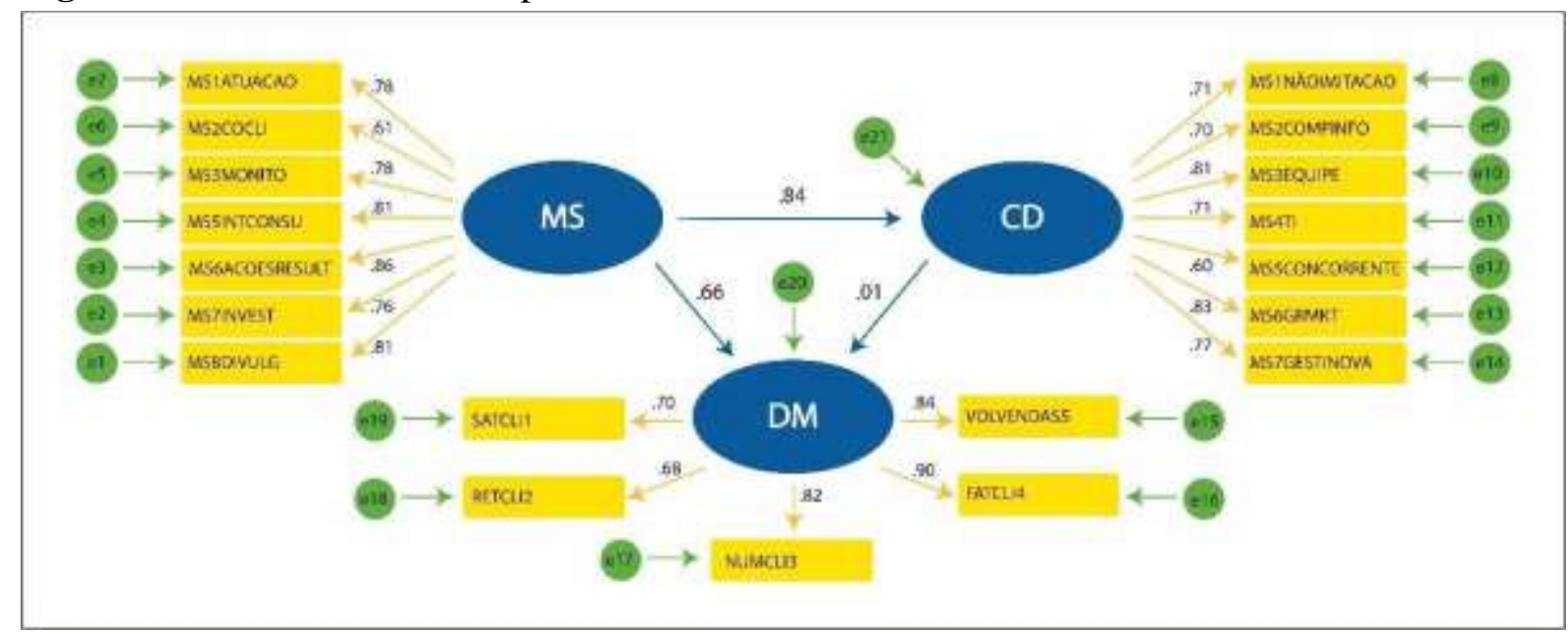

Source: Research data, 2018.

The indicators of the model's general fit were 2.537 for the value of CMIN/DF, 0.877 for CFI, and 0.104 for RMSEA, and show that the adjustment of the model is not ideal, but is reasonable, as recommended by Hair et al. (2009).

The last step was to analyze the research hypotheses and significance tests. The coefficients calculated for the relationships between the constructs predicted in hypotheses $\mathrm{H} 1$ and $\mathrm{H} 2$ were accepted (p-value $=0.000$ ). Thus, we confirmed that SM influences DC and MP. However, hypothesis H3 was rejected, showing that DC does not affect MP (see Table 4). 
Table 4 - Results of significance tests of the research models

\begin{tabular}{cccccccc}
\hline Hypothesis & $\begin{array}{c}\text { Dependent } \\
\text { variable }\end{array}$ & & $\begin{array}{c}\text { Independent } \\
\text { Variable }\end{array}$ & Estimate & $\boldsymbol{p}$-value & $\boldsymbol{C R}$ & Status \\
\hline H1 & SM & $--->$ & DC & .842 & 0.000 & 6.409 & Accepted \\
H2 & SM & $--->$ & MP & .657 & 0.000 & 3.646 & Accepted \\
H3 & DC & $--->$ & MP & .007 & 0.691 & 0.420 & Rejected \\
\hline
\end{tabular}

Source: Research data, 2018.

To calculate mediation, we used the criteria indicated by Hair et al. (2009): (i) the independent and dependent variables must have a significant correlation. In this case, the correlation between SM and MP was 0.624, with p-value of 0.000, thus meeting the first item; (ii) the independent variable must have a significant correlation with the mediating variable being tested. In this case, the correlation between SM and DC was 0.743 , with p-value of 0.000 , also meeting the second item; and (iii) the mediating variable must have a significant correlation with the dependent variable. In this test, DC and MP showed a correlation of 0.528 with p-value of 0.000 , meeting the third item.

The following step was to calculate the effect relationship between the independent and dependent variables and, subsequently, recalculate with the inclusion of the mediating variable. We did the first calculation with the AMOS® software, using the SM and MP constructs. Path coefficient was 0.66 , significant at the 0.001 level. Then, we included construct DC, and redid the calculations. The path coefficient between SM and MP did not change, remaining at 0.66 , significant at the 0.001 level, as shown in Figure 3. Hair et al. (2009) state that if the relationship between the independent and dependent variables remains significant and unchanged, with the inclusion of the mediating variable, there is no mediation. Therefore, DC mediation between SM and MP was not supported.

To confirm the absence of mediation, we conducted a second calculation with the Macro Process for SPPS, by Andrew F. Hayes. We included the independent variable SM, the dependent variable MP, and the mediating variable DC. Table 5 shows the result.

Table 5 - Results of significance tests of the mediating models

\begin{tabular}{|c|c|c|c|c|c|c|}
\hline \multicolumn{7}{|c|}{ Model summary } \\
\hline $\mathrm{R}$ & R-sq & MSE & $\mathrm{F}$ & df1 & $\mathrm{df} 2$ & $\mathrm{p}$ \\
\hline 0.631 & 0.398 & 245.253 & 46.310 & 2.000 & 140.000 & 0.000 \\
\hline \multicolumn{7}{|c|}{ Model } \\
\hline & coef & se & $\mathrm{t}$ & $\mathrm{p}$ & LLCI & ULCI \\
\hline Constant & 22.878 & 4.691 & 4.878 & 0.000 & 13.605 & 32.152 \\
\hline SM & 0.499 & 0.095 & 5.272 & 0.000 & 0.312 & 0.686 \\
\hline DC & 0.147 & 0.100 & 1.469 & 0.144 & -0.051 & 0.345 \\
\hline
\end{tabular}

Note: Dependent variable: MP

Source: Research data, 2018. 
The model's explanation is $39.8 \%$. The table also shows that SM accounts for $49.9 \%$ of MP, significant at 0.000 . The latent variable DC explains $14.7 \%$ of MP. However, such explanation is not significant, thus we conclude that DC does not contribute to the model's explanation. Thus, as the previous result already showed, $\mathrm{H} 4$ was rejected, since DC do not mediate the relationship between SM and MP.

\subsection{Discussion}

The results of this study indicate that SM generates DC and also MP, in our sample of MSMEs; however, when SM is mediated by DC, there is no influence on MP, that is, the use of SM does not generate MP indirectly, through DC. The participating companies confirm the observations of Teece et al. (1997), that firms exploit their internal and external competencies to face the changing environment. Therefore, SM generates DC, which confirms H1 statistically. The results are close to those found by Lam et al. (2016), who considered that social media enables the improvement of dynamic routines, achieving better performance.

There are indications that the analyzed companies should internalize the use of social media to get to know and relate better with their stakeholders. The immersion of companies in SM can change their relationship with stakeholders, as they provide a platform for sharing experience, accumulating knowledge, and organizational learning (Nguyen et al., 2015; Lam et al., 2016). MSMEs in our sample, in the year 2017, timidly increased experience sharing, the use of information to know customers, and new actions, based on SM information.

In the SM construct, small companies have higher performance means in social media, followed by micro companies. This result is close to that found by Braojos-Gomez et al. (2015), who showed, in their empirical analysis, that the competence in social networks is more important for smaller companies. One explanation regards their smaller portfolio of financial resources, so that media capabilities become more important for them than for larger companies.

The results of the statistical calculations supported hypothesis H2 - "the use of SM affects performance". This result reflects and confirms those by Venkatraman and Ramanujam (1986) and Callado et al. (2008), regarding the identification and measurement of the use of SM in companies to increase performance. Our findings are similar to those of Lombardi et al. (2010) and Santos et al. (2014), indicating that when companies operate in competitive and uncertain environments, managers' decisions are important to achieve higher profitability, sales growth, and win new customers. Our study shows that, although Santa Catarina's MSMEs are concerned with increasing income and retaining customers, it is still possible to improve their performance.

Some studies confirm this view, by showing that to reach MP, companies need to develop $\mathrm{DC}$, be able to attract new customers, retain current ones, increase profitability, as well as market 
share, besides offering higher quality, attractive prices, and customized service (Christensen, 1997; OECD, 2005; Tidd et al., 2005; Tigre, 2006; Chesbrough, 2012; Teece et al., 2014).

The findings of this study are similar to those of Braojos-Gomez et al. (2015), when suggesting that the capacity of IT infrastructure, the pressure of social competitors, marketing management and innovation management are essential mechanisms, through which small companies learn to develop competence in social networks.

Hypothesis H3 - "DC positively affect market performance", that is, DC generate MP, was not supported, contrary to the findings by Wardaya et al. (2019), which received empirical support. The results of our research reinforce that MSMEs must develop the essential mechanisms through which they can learn to achieve competences in social networks (Braojos-Gomez et al., 2015). The results also showed that the capacity of IT infrastructure, for example, did not increase or decrease in 2017, the pressure from social competitors had a little increase, and marketing management and innovation management increased slightly, which suggests that companies need an internal commitment and investment in social media marketing to improve their performance.

The results are also close to Teece et al.'s statement (2014) that wealth creation in regimes of high technological change depends, largely, on the company's technological and organizational capacity, and on management processes. The companies studied also have difficulty in changing SM data into valuable personal knowledge, and exploiting these insights to add value to customers (Wieneke \& Lehrer, 2016). Thus, we could not confirm the statistical validity of the hypothesis, since these companies still need to promote their DC.

Theory shows that DC enable developing market differentials in the face of competition (Eisenhardt \& Martin, 2014). Our results indicate that MSMEs in Santa Catarina seek such differentials, but there is still room for improvement. This is clear when we analyze the average score of the variable "work to prevent SM resources from being identified/imitated", as well as the variable "with the use of SM, pressure by competitors". Therefore, companies seem not to use or ignore DC as a resource to improve their MP (Eisenhardt, \& Martin, 2014). Moreover, the relationship between DC and competitive advantage is based on the company's ability to create, configure, integrate and combine its resources (Eisenhardt, \& Martin, 2014), and this depends on the organizations' maturity, as it affects MP (Barra \& Ladeira, 2017; Barney \& Hesterly, 2007).

Finally, Barra and Ladeira (2017) and Barney and Hesterly (2007) state that different strategies (rare, inimitable, valuable, and organized capabilities and resources) can create value for the customer, and lead to a superior performance. This statement contributes to understanding that the studied MSMEs still need to absorb and learn from their DC. 


\section{Conclusion}

The main objective of the study was to analyze the impact of the use of social media on dynamic capabilities and market performance of micro, small and medium-sized companies operating in the state of Santa Catarina, Brazil. In order to test the relationship between the constructs, we applied a survey to companies' managers in that state and received 143 answers. The structure of the study integrates and synthesizes concepts and results of the field research, which show that SM provides DC, SM generates MP, but DC do not lead to MP.

SM capabilities seem to be more important for small companies than for large ones. However, due to the restriction of financial resources, the model demonstrates that MSMEs need to develop more DC, so that the effectiveness of SM turns into MP. Thus, our findings confirm that companies have difficulties regarding IT infrastructure capacity, pressure from social competitors, and marketing and innovation management capabilities. These are essential mechanisms, through which companies learn to develop competence in social networks (Braojos-Gomez et al., 2015).

The study also shows that SM competence is more important for small companies. All mentioned mechanisms (capacity of IT infrastructure, social pressure from competitors, management of marketing and innovation) are crucial antecedents, through which small firms learn to develop a competence in social networks, although we did not test their order of importance (Braojos-Gomez et al., 2015).

According to the study, MSMEs need to improve their DC, that is, seek to absorb information from the digital world and change SM data into personal and valuable knowledge, in order to exploit them to add value to customers. It also contributed by indicating that wealth creation in environments of high technological change depends, largely, on the firm's technological, organizational and managerial capacity.

Wardaya et al. (2019) found that marketing and market orientation skills are crucial to improve performance; however, our results do not support the influence of dynamic capabilities' mediation on the relationship between the use of social media and market performance. There is no point in being present on the networks without actually adopting planned strategies for digital marketing.

We conclude that MSMEs in the State of Santa Catarina are in a ripening process regarding the use of social media to generate dynamic capabilities and market performance. They show progress, but they can still improve, by developing DC that enable better performance. It is precisely in achieving performance, based on DC, that lie the biggest challenges, since managers, through their decisions, are responsible for internal development and market conquest. 


\section{Research limitations and suggestions for future studies}

Although the proposed model explains issues related to MSMEs in the State of Santa Catarina, there may be metric factors in the SM of these companies, or perceptions from their stakeholders, which affect DC and the MP of SM. It is still necessary to consider that this study focused on the perceptions of companies' managers; therefore, there is a motivation for researchers to expand data collection to employees and customers, with the possibility of using secondary data on companies' social media. Future studies may also increase the number of companies in the sample. Another issue concerns the fact that the study covered micro, small and medium-sized companies. Perhaps, with just one category, the results would be different. Finally, it is important to carry out additional research to understand how social media capabilities influence the performance of large companies' innovations, adding market capacity as a construct.

\section{References}

Ahani A., Rahim, N. Z. A., \& Nilashi M. (2017). Forecasting social CRM adoption in SMEs: A combined SEM-neural network method. Computers in Human Behavior, 75, 560-578. https://doi.org/10.1016/j.chb.2017.05.032

Barney, J. (1991). Firm resources and sustained competitive advantage. Journal of Management, 17(1), 99-120. https://doi.org/10.1177/014920639101700108

Barney, J.B, \& Hesterly, W.S. (2007). Administração Estratégica e Vantagem Competitiva: casos brasileiros. São Paulo: Pearson.

Barra, G. M. J., \& Ladeira, M. B. (2017). Modelo de maturidade para processos de certificação no sistema agroindustrial do café. REGE-Revista de Gestão, 24(2), 134-148. https://doi.org/10.1016/j.rege.2017.03.004

Braganza, A., Brooks, L., Nepelski, D., Ali, M., \& Moro, R. (2017). Resource management in big data initiatives: Processes and dynamic capabilities. Journal of Business Research, 70, 328337. https://doi.org/10.1016/j.jbusres.2016.08.006

Braojos-Gomez, J., Benitez-Amado, J., \& Llorens-Montes, F. J (2015). How do small firms learn to develop a social media competence? International Journal of Information Management, 35(4), 443-458. https://doi.org/10.1016/j.ijinfomgt.2015.04.003

Callado, A. A. C., Callado, A. L. C., \& Andrade, L. P. (2008). Padrões de utilização de indicadores de desempenho não-financeiros: um estudo exploratório nas empresas de Serra Talhada/PE. ABCustos, 3(2), 1-26.

Castells, M. (2003). A galáxia da Internet. Rio de Janeiro: Jorge Zahar.

Chenhall, R. H., \& Moers, F. (2015). The role of innovation in the evolution of management accounting and its integration into management control. Accounting, Organizations and Society, 47, 1-13. https://doi.org/10.1016/j.aos.2015.10.002 
Chesbrough, H. (2012). Inovação aberta: Como criar e lucrar com a tecnologia. Porto Alegre: Bookman.

Christensen, C. M. (1997). The innovator's dilemma: When new technologies cause great firms to fail. Boston, MA: Harvard Business School Press.

Eisenhardt, K. M., \& Martin, J. A. (2000). Dynamic capabilities: what are they? Strategic Management Journal, 21(10/11), 1105-1121. https://doi.org/10.1002/1097$\underline{0266(200010 / 11) 21: 10 / 11<1105:: A I D-S M J 133>3.0 . C O ; 2-E}$

Eisenhardt, K. M., \& Martin, J. A. (2014). O que são as capacidades dinâmicas? In D. P. Lacerda, R. Teixeira, J. Antunes, \& Corcini, S. L. H., Neto (Eds.). Estratégia Baseada em Recursos - 15 artigos clássicos para sustentar Vantagens Competitivas (pp. 211-240). Porto Alegre: Bookman.

Erevelles, S., Fukawa, N., \& Swayne, L. (2016). Big Data consumer analytics and the transformation of marketing. Journal of Business Research, 69(2), 897-904. https://doi.org/10.1016/j.jbusres.2015.07.001

Gupta, M., \& George, J. (2016). Toward the development of a big data analytics capability. Information \& Management, 53(8), 1049-1064. https://doi.org/10.1016/j.im.2016.07.004

Hair, J. F., Jr., William, B., Babin, B., \& Anderson, R. E. (2009). Análise multivariada de dados (6a ed.). Porto Alegre: Bookman.

Instituto Brasileiro de Geografia e Estatística [IBGE]. Agência de Notícias. PNAD Contínua TIC 2017: Internet chega a três em cada quatro domicilios do país. Recuperado de https://agenciadenoticias.ibge.gov.br/agencia-sala-de-imprensa/2013-agencia-denoticias/releases/23445-pnad-continua-tic-2017-internet-chega-a-tres-em-cada-quatrodomicilios-do-pais.

Jantunen, A., Tarkiainen, A., Chari, S., \& Oghazi, P. (2018). Dynamic capabilities, operational changes, and performance outcomes in the media industry. Journal of Business Research, 89, 251-257. https://doi.org/10.1016/j.jbusres.2018.01.037

Jenkins, H. (2006). Convergence culture: Where old and new media collide. New York: New York Univ. Press.

Lam, H. K. S., Yeung, A. C. L., \& Cheng, T.C. E. (2016). The impact of firms' social media initiatives on operational efficiency and innovativeness. Journal of Operations Management, 47, 28-43. https://doi.org/10.1016/j.jom.2016.06.001

Li, L., Su, F., Zhang, W., \& Mao, J. (2017). Digital transformation by SME entrepreneurs: A capability perspective. Information Systems Journal, 28(6), 1129-1157.

https://doi.org/10.1111/isj.12153

Lin, Y., \& Wu, L. Y. (2014). Exploring the role of dynamic capabilities in firm performance under the resource-based view framework. Journal of Business Research, 67(3), 407-413. https://doi.org/10.1016/j.jbusres.2012.12.019 
Liu, Y., Deng, S., Hu, F., \& Chen, X. (2015). The impacts of unique service resources and habit on e-service loyalty in a highly competitive market. Journal of Systems and Information Technology, 17(4), 336-350. https://doi.org/10.1108/JSIT-06-2015-0050

Lombardi, M. S., Bertoni, C. E., Nozawa, G. I., Bellato, T. \& Grandi. L. A. (2010) A percepção da incerteza e o desempenho operacional da indústria brasileira de 2007 a 2009. Future Studies Research Journal: Trends and Strategies, 2(2), 56-82.

Martynow, A., \& Shafti, N. (2016). Long-term performance of firms: a review and research agenda. Journal of Strategy and Management, 9(4), 429-448. https://doi.org/10.1108/JSMA-02$\underline{2015-0016}$

Mention, A., Barlatier, P-J., \& Josserand, E. (2019). Using social media to leverage and develop dynamic capabilities for innovation. Technological Forecasting and Social Change, 144, 242250. https://doi.org/10.1016/j.techfore.2019.03.003

Nguyen, B., Yu, X., Melewar, T. C., \& Chen, J. (2015). Brand innovation and social media: knowledge acquired from social media, market orientation, and the moderating role of social media strategic capability. Industrial Marketing Management, 51, 11-25. https://doi.org/10.1016/j.indmarman.2015.04.017

Organization for Economic Cooperation and Development [OECD] (2005). Modernizing Government: The way forward. Paris: OECD Publishing.

Peteraf, M. A. (1993). The cornerstones of competitive advantage: a resource-based view. Strategic Management Journal, 14(3), 179-191. https://doi.org/10.1002/smj.4250140303

Pinho, J. C., \& Prange, C. (2016). The effect of social networks and dynamic internationalization capabilities on international performance. Journal of World Business, 51(3), 391-403. https://doi.org/10.1016/j.jwb.2015.08.001

Pogrebnyakov, N. (2017). A cost-based explanation of gradual, regional internationalization of multinationals on social networking sites. Management International Review, 57(1), 37-64. https://doi.org/10.1007/s11575-016-0281-z

Porter, M.E. (1980). Competitive Strategy: Techniques for Analyzing Industries and Competitors. New York: Free Press.

Saavedra F. U., Andreu J. L., \& Criado, J.R. (2016). The impact of social media marketing on the relationship among dynamic capabilities and performance. In C. Campbell, \& J. J. Ma (Eds.). Looking forward, looking back: Drawing on the past to shape the future of marketing. Proceedings of the 2013 World Marketing Congress. Cham: Springer.

Santos, N. M., Bronzo, M., Oliveira, M. P. V., \& Resende, P. T. V. (2014). Cultura organizacional, estrutura organizacional e gestão de pessoas como bases para uma gestão orientada por processos e seus impactos no desempenho organizacional. Brazilian Business Review, 11(3), 106-129. https://doi.org/10.20397/2177-6652/2017.v17i1.915

Serviço Brasileiro de Apoio às Micro e Pequenas Empresas [SEBRAE] (2017). Anuário do trabalho nos pequenos negócios 2015 (8a ed.). São Paulo:DIEESE. 
Teece, D. J., Pisano, G., \& Shuen, A. (1997) Dynamic capabilities and strategic managment. Strategic Managment Journal, 18(7), 509-533. https://doi.org/10.1002/(SICI)10970266(199708)18:7<509::AID-SMJ882>3.0.CO;2-Z

Teece, D., Pisano, G., \& Shuen, A. (2014). Capacidades dinâmicas e gestão estratégica. In D. P. Lacerda, R. Teixeira, J. Antunes, \& Corcini, S. L., Neto (Eds.) Estratégia Baseada em Recursos. Porto Alegre: Bookman.

Telles, A. (2011). A revolução das mídias sociais: Cases, conceitos, dicas e ferramentas. São Paulo: M. Books.

Tidd, J., Bessant, J., \& Pavitt, K. (2005). Managing innovation: Integrating technological, market and organizational change (3 ed.). Chichester: John Wiley \& Sons.

Tigre, P. B. (2006). Gestão da inovação. Rio de Janeiro: Campus.

Venkatraman, N., \& Ramanujam, V. (1986). Measurement of business performance in strategy research: a comparison of approaches. Academy of Management Review, 11(4), 801-814. https://doi.org/10.5465/amr.1986.4283976

Wamba, S. F., Gunasekaran, A., Akter, S., Ren, S. J., Dubey, R., \& Childe, S. J. (2017). Big data analytics and firm performance: Effects of dynamic capabilities. Journal of Business Research, 70, 356-365. https://doi.org/10.1016/j.jbusres.2016.08.009

Wang, C. L., \& Ahmed, P. K. (2007) Dynamic capabilities: a review and research agenda. International Journal of Management Reviews, 9(1), 31-51. https://doi.org/10.1111/j.1468$\underline{2370.2007 .00201 . x}$

Wang, Z., \& Kim, H. G. (2017). Can social media marketing improve customer relationship capabilities and firm performance? Dynamic capability perspective. Journal of Interactive Marketing, 39, 15-26. https://doi.org/10.1016/j.intmar.2017.02.004

Wardaya, A., Idris, S., So, I. G., \& Bandur, A. (2019). Mediating effects of digital marketing on dynamic capability and firm performance: Evidence from small and medium-sized enterprises (SMEs) in Indonesia. International Journal of Recent Technology and Engineering (IJRTE), 8(5C), 692-696. DOI:10.35940/ijeat.E1098.0585C19

Wieneke, A., \& Lehrer, L. (2016). Generating and exploiting customer insights from social media data. Electronic Markets, 26(3), 245-268. doi:10.1007/s12525-016-0226-1

Zavattaro, S. M., \& Daspit, J. J. (2016). A grounded theoretical approach to understanding innovation in destination marketing organizations. Journal of Vacation Marketing, 22(4), 349364. doi:10.1177/1356766715623826

Zollo, M., \& Winter, S. G. (2002). Deliberate learning and the evolution of dynamic capabilities. Organization Science, 13(3), 339-351. https://doi.org/10.1287/orsc.13.3.339.2780. 


\section{Appendix 1 - Questionnaire}

Tick the main sector of activity of your company:

Commerce

Industry

Provision of services

Indicate the number of employees in your company:

Indicate the main city of your company:

Regarding the use of social media by your company in 2017 , tick each of the options, by choosing from " $1=$ no use" to "5= maximum use".

Facebook

Google+

Instagram

LinkedIn

Messenger

Pinterest

Skype

Snapchat

Telegram

Tumblr

Twitter

WhatsApp

You Tube

Other (please mention):

For the following questions, position the cursor and click on the bar, according to your perception regarding the company, being $0=$ reduced $10 \%$ or more, $50=$ neither reduced nor increased, and $100=$ increased $10 \%$ or more.

In order to answer, please consider the year 2017:

The activity of my company in social media consisted of:

Using the information from social media to know my customer:

Monitoring our company's social media:

Planning the strategies for our company's social media:

Firm's interaction with the consumer, on social media:

Actions performed by our firm from social media results:

Our company's investment in social media:

Promotion of our products or services on social media:

Work to ensure our social media resources from being easily identified or imitated by competitors:

Sharing of social media information among our company's sectors, to reach an integrated management:

With the use of social media, reorganization or building a business team or the company's internal skills:

With the use of social media, our firm's IT infrastructure capacity:

With the use of social media, pressure from our competitors:

With the use of social media, our efforts for marketing management:

With the use of social media, our efforts for innovation management:

With the use of social media, our responsiveness to changes in the competitive environment:

Our customers' level of satisfaction:

Level of retention of customers:

Number of new customers:

Our company's income:

Sales volume of our company: 